\title{
Effective slip law for general viscous flows over an oscillating surface
}

\author{
Andro Mikelić ${ }^{* \dagger}$ \\ Université de Lyon, CNRS UMR 5208, \\ Université Lyon 1, Institut Camille Jordan,
}

43, blvd. du 11 novembre 1918, 69622 Villeurbanne Cedex, FRANCE

Šarka Nečasová $\ddagger \S$

Institute of Mathematics of the Academy of Sciences

of the Czech Republic, Žitná 25, 11567 Praha 1,

Prague, CZECH REPUBLIC

Maria Neuss-Radu 9

Mathematics Department, University of Erlangen-Nuremberg, Cauerstr. 11, 91058 Erlangen, GERMANY

April 30, 2013

\begin{abstract}
We consider the non-stationary three dimensional viscous flow in a bounded domain, with the lateral surface containing microscopic surface irregularities. Under the assumption of a smooth flow in the domain without roughness, we prove that there is a smooth solution to a problem with the rough boundary. In the papers by Jäger and Mikelić the friction law was obtained as a perturbation of the Poiseuille flows. Here situation is more complicated. Nevertheless, after studying the corresponding boundary layers and using results on solenoidal vector fields in domains with rough boundaries, we obtain rigorously the Navier friction condition. It is valid when the size and amplitude of the imperfections tend to zero. Furthermore, the friction matrix in the law is determined through a family of auxiliary boundary-layer type problems. Effective equations approximate velocity at order $\mathrm{O}(\varepsilon)$ in the $H^{1}$-norm, uniformly in time, and $\mathrm{O}\left(\varepsilon^{3 / 2}\right)$ in the $L^{2}$-norm, also uniformly in time. Approximation for the pressure is $\mathrm{O}\left(\varepsilon^{3 / 2}\right)$ in the $L_{l o c}^{2}$-norm.
\end{abstract}

Keywords Boundary with irregularities, roughness, boundary homogenization, Navier's boundary condition

AMS subject classifications. 76D05, 35B27, 76Mxx, 35Qxx

\footnotetext{
${ }^{*}$ The research of A.M. was partially supported by the Programme Inter Carnot Fraunhofer from BMBF (Grant 01SF0804) and ANR. He is grateful to the Ruprecht-Karls-Universität Heidelberg and the Heidelberg Graduate School of Mathematical and Computational Methods for the Science (HGS MathComp) for giving him good working conditions through the W. Romberg Guest Professorship 2011-2013.

${ }^{\dagger}$ E-mail: Andro.Mikelic@univ-lyon1.fr

${ }^{\ddagger}$ The research of $\breve{S}$. N. acknowledges the support of the GAČR (Czech Science Foundation) project P201-13-00522S in the general framework of RVO: 67985840.

${ }^{\S}$ E-mail: matus@math.cas.cz

๑E-mail: maria.neuss-radu@am.uni-erlangen.de
} 


\section{Introduction}

Physics of the laminar viscous fluid flow imposes the no-slip condition $\mathbf{v}=0$, at an immobile smooth solid boundary. Since the fluid cannot penetrate the solid, we have the non-penetration condition $\mathbf{v} \cdot \mathbf{n}=0$. The absence of slip is not intuitive and corresponds to the experimentally observed situation at a smooth solid wall (see [39]). Contesting it, goes back to Navier himself (see [37]). Kinetic-theory calculations confirmed Navier's slip law, but the slip length is proportional to the mean free path divided by the continuum length (see [39]) and hence zero for most practical proposes.

In many cases of practical significance the boundary contains asperities. Examples are complex sea bottoms of random roughness and artificial bodies with periodic distribution of small bumps. Description of an oscillating rough boundary requires a big quantity of data and makes a numerical solution of the related flow problems complex and costly. For computational purposes, the original oscillating rough boundary is replaced by an artificial non-oscillatory smooth boundary, close to the original one. The equations are solved in a new domain. This way the rough boundary is avoided, but the boundary conditions at the artificial boundary are to be determined. For the incompressible flows, it is clear that the non-penetration condition should be kept. To the contrary it is not clear if the no-slip condition in the tangential directions could be still used. In the literature, it is frequently supposed that the shear stress is some function $F$ of the tangential velocity. Such functional relations are called the wall laws.

There is a huge literature on the asymptotic behavior of PDEs in the presence of oscillating boundaries. For more information one could consult the review paper [35]. Here we concentrate on flow problems over rough boundaries.

Early mathematical work on the Couette flow over a rough plate and applications to drag reduction is due to Amirat, Simon and collaborators in [4], [5] and [6]. Their approach has similarities with the boundary layers theory from [3] and [32] and they concentrate on small Reynolds numbers.

Modeling and computational of flow problems over rough surfaces was undertaken by Pironneau and collaborators in the papers [36], [1] and [2]. The paper [36] considers the flow over a rough surface and the flow over a wavy sea surface. In the paper [2] the stationary incompressible flow at high Reynolds number $\mathbf{R e} \sim \frac{1}{\varepsilon}$ over a periodic rough boundary, with the roughness period $\varepsilon$, is considered. An asymptotic expansion is constructed and effective wall laws are obtained. A numerical validation is presented, but there are no mathematically rigorous convergence results. The most renown wall law is the Navier slip condition from [37], and its formal derivations are already known in the engineering literature (see e.g. [33]).

First mathematically rigorous derivation of Navier's slip condition is due to Jäger and Mikelić in [28]. It is based on the approach from [25] and [27] in obtaining the interface laws between porous media and an unconfined fluid flow. The auxiliary problems, used to calculate the effective coefficients for the Beavers and Joseph interface law, were slightly modified and used in obtaining the constants in Navier's slip law. In [28] justification of the approximation in the case of the stationary Navier-Stokes equations with flow governed by a pressure drop, was undertaken. The Couette flow around rough boundary (riblets) was studied in [30]. Rigorous estimates lead to conclusion that the riblets reduce significantly tangential drag, which may explain their presence on the skin of Nektons. 
For generalization to quasi-geostrophic flows we refer to [9] and references therein.

All these results were obtained for periodic rough boundaries. In fact realistic natural rough boundaries are random and for study of flows in such geometries we refer to the works of Gérard-Varet and collaborators [8], [23] and [24].

In the above work, the results on the Navier slip condition were obtained by looking at the perturbations of Couette and Poiseuille flows. Such particular setting appears in many applications and allows avoiding complications with the incompressibility condition. However for a general incompressible viscous flow situation is more complicated. We need pressure estimates in presence of an oscillating boundary. Since we have the non-penetration condition, the wall law for the pressure is not required. We note that in the case of the interface unconfined fluid/porous medium one obtains the pressure jump as one of effective interface laws. For more details we refer to [29], [34] and [14].

Conversely, there have been several attempts in the literature to provide a rigorous justification of the no-slip boundary conditions based on the idea that the physical boundary is never smooth but contains small asperities that drive the fluid to rest. Results of this type have been shown by Casado-Diaz et al. [17] in the case of periodically distributed asperities and later extended to the general case in [12]. It is worth noting that these results are not contradictory but reflect two conceptually different approaches in mathematical modeling of viscous fluids. Recent developments in macrofluidic and nanofluidic technologies have renewed interest in the slip behavior that may become significant in the small spatial scales even for a relatively small Reynolds number (cf. Priezjev and Troian [40]). In article [13] a family of solutions to the evolutionary Navier-Stokes system supplemented with the complete slip boundary conditions on domains with rough boundaries was considered and a complete description of the asymptotic limit by means of $\Gamma$-convergence arguments was given. As a result, a general class of boundary conditions was identified in the case of equi-Lipschitz domain. The extension of previous result can be found in [10], where authors considered domains with arbitrary non-periodical crystalline boundaries and general non-smooth periodical boundaries. For even larger class of boundary conditions, homogenization of the Navier-Stokes equation in a channel with a rough boundary was considered in [21]. They generalized their previous results from [24, 8], give error estimates for this homogenized no-slip boundary condition of Navier type and find a more accurate effective boundary condition of Navier type. For the asymptotic behavior of viscous fluid satisfying Navier's condition on a slightly rough boundary we refer to recent articles $[15,16]$. Finally, the wall law for very rough surface was investigated in [7].

The goal of this paper is to obtain rigorously Navier's slip condition in the general situation. In Section 2 we introduce the problem by defining precisely the geometry, recall the result on solenoidal vector fields from [11] and formulate the microscopic problem. In Subsection 2.3 the main result on approximation by an effective slip law is formulated. Zeroth order approximation, constructed in Section 3, requires that we prove existence of a smooth solution to a microscopic problem, close to the solution to the problem without roughness. It is proved in Theorem 1, which is of independent interest. The next order correction, leading to Navier's slip condition, is constructed in Section 4. Proof of the main result is in Section 5. It requires the proof of existence of smooth solutions to a 3D incompressible Navier-Stokes system with the mixture of slip, no-slip and periodic boundary conditions. Finally, there is Appendix 6, where construction of the very weak solution to the Oseen problem is recalled. 


\section{Statement of the problem and main result}

\subsection{Definition of the geometry}

We consider a viscous flow taking place in a domain $\Omega^{\varepsilon}$ having a rough boundary. It is described as follows.

Let $\Omega=(0, L)^{3}$. Its bottom and top boundaries are $\Sigma=(0, L)^{2} \times\{0\}$ and $\Sigma_{L}=$ $(0, L)^{2} \times\{L\}$, respectively. Adjacent to $\Sigma$ there is the thin layer $R^{\varepsilon}$, having a rapidly oscillating boundary, which is defined by help of the following map.

Let $\varepsilon>0$ be such that $L / \varepsilon \in \mathbb{N}$. Let

$$
\Upsilon:(0,1)^{2} \rightarrow \mathbb{R}, \quad\left(y_{1}, y_{2}\right) \mapsto \Upsilon\left(y_{1}, y_{2}\right)
$$

be a Lipschitz surface, with Lipschitz constant $L_{\Upsilon}$, 1-periodic with respect to $y_{1}$ and $y_{2}$, and satisfying

$$
0 \leq \Upsilon\left(y_{1}, y_{2}\right) \leq b_{3}<1, \quad\left(y_{1}, y_{2}\right) \in(0,1)^{2} .
$$

The canonical cell of roughness (the canonical hump) $Y \subset Z=(0,1)^{3}$ is $Y=\{y \in Z \mid$ $\left.\Upsilon\left(y_{1}, y_{2}\right)<y_{3}<1\right\}$, the corresponding infinite layer $\mathcal{L}=\cup_{\left\{k \in \mathbb{Z}^{2}\right\}}\left(Y+\left(k_{1}, k_{2},-1\right)\right)$, and the standard rough boundary

$$
\mathcal{B}=\left\{\left(y_{1}, y_{2}\right) \in(0,1)^{2}, y_{3}=\Upsilon\left(y_{1}, y_{2}\right)-1\right\} .
$$

For $0<\varepsilon<<1$ the layer of roughness $R^{\varepsilon}$ is now given by

$$
R^{\varepsilon}=\varepsilon \mathcal{L} \cap\left((0, L)^{2} \times(-\varepsilon, 0)\right),
$$

and its rough part of the boundary is

$$
\mathcal{B}^{\varepsilon}=\left\{x \in \mathbb{R}^{3}:\left(x_{1}, x_{2}\right) \in(0, L)^{2}, x_{3}=\varepsilon\left(\Upsilon\left(\frac{x_{1}}{\varepsilon}, \frac{x_{2}}{\varepsilon}\right)-1\right)\right\} .
$$

Thus $\mathcal{B}^{\varepsilon}$ consists of a large number of periodically distributed humps of characteristic length and amplitude of order $\varepsilon$, small compared with the characteristic length of the macroscopic domain. For a given $T>0$ we set

$$
Q_{T}=\Omega \times(0, T), \quad \Omega^{\varepsilon}=\Omega \cup \Sigma \cup R^{\varepsilon} \quad \text { and } \quad \partial \Omega_{l a t}=\partial \Omega^{\varepsilon} \backslash\left(\mathcal{B}^{\varepsilon} \cup \Sigma_{L}\right) .
$$

We refer to Figure 1 for the graphical representation. $\mathbf{n}$ is the outer unit normal to $\partial \Omega^{\varepsilon}$. We will work in the following functional space

$$
V\left(\Omega^{\varepsilon}\right)=\left\{\varphi \in H^{1}\left(\Omega^{\varepsilon}\right): \varphi=0 \text { on } \Sigma_{L} \cup \mathcal{B}^{\varepsilon}, \varphi \text { is L-periodic in } x_{1}, x_{2}\right\} .
$$

Every element of $V\left(\Omega^{\varepsilon}\right)$ is extended by zero to $(0, L)^{2} \times(-\varepsilon, 0) \backslash R^{\varepsilon}$.

For domains $\Omega^{\varepsilon}$ defined above, the following representation theorem holds:

Theorem 1. Let $\Omega^{\varepsilon} \subset \mathbb{R}^{3}$ be defined as in Section 2.1, and let $g \in L^{2}\left(\Omega^{\varepsilon}\right)$ be such that $\int_{\Omega^{\varepsilon}} g d x=0$. Then the function $g$ admits the representation

$$
g=\operatorname{div} \mathbf{F}^{\varepsilon} \quad \text { in } \Omega^{\varepsilon},
$$

with $\mathbf{z}^{\varepsilon} \in H^{1}\left(\Omega^{\varepsilon}\right)^{3}, \mathbf{z}^{\varepsilon}=0$ on $\Sigma_{L} \cup \mathcal{B}^{\varepsilon}, \mathbf{z}^{\varepsilon}$ is $L$-periodic in $x_{1}$ and $x_{2}$, and the following estimate holds

$$
\left\|\mathbf{z}^{\varepsilon}\right\|_{H^{1}\left(\Omega^{\varepsilon}\right)} \leq C\|g\|_{L^{2}\left(\Omega^{\varepsilon}\right)} .
$$

Proof. For the proof, see e.g. Proposition 5.2 in [11]. 

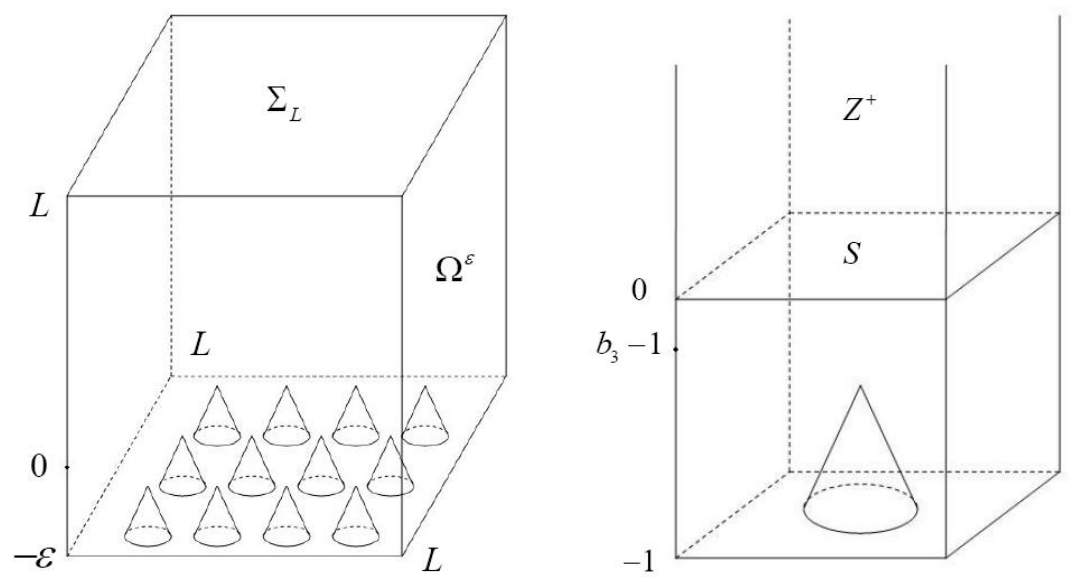

Figure 1: The domain $\Omega^{\varepsilon}$, and the boundary layer cell $Z_{b l}$.

\subsection{The microscopic equations}

Let $\mathbf{f} \in C^{\infty}\left(\left([0, L]^{2} \times[-\varepsilon, L]\right) \times[0, T]\right)^{3}$, supp $\mathbf{f} \subset\left([0, L]^{2} \times[-\varepsilon, L]\right) \times(0, T], \mathbf{f}$ is periodic in $\left(x_{1}, x_{2}\right)$ with period $L$. We consider the following problem:

$$
\begin{aligned}
& \frac{\partial \mathbf{u}^{\varepsilon}}{\partial t}+\operatorname{div}\left(\mathbf{u}^{\varepsilon} \otimes \mathbf{u}^{\varepsilon}\right)-\frac{1}{\mathbf{R e}} \operatorname{div}\left(2 D\left(\mathbf{u}^{\varepsilon}\right)-p^{\varepsilon} I\right)=\mathbf{f} \quad \text { in } \Omega^{\varepsilon} \times(0, T), \\
& \operatorname{div} \mathbf{u}^{\varepsilon}=0 \quad \text { in } \Omega^{\varepsilon} \times(0, T), \\
& \mathbf{u}^{\varepsilon}=0 \quad \text { on }\left(\mathcal{B}^{\varepsilon} \cup \Sigma_{L}\right) \times(0, T), \\
& \mathbf{u}^{\varepsilon} \quad \text { is periodic in }\left(x_{1}, x_{2}\right) \text { with period } L, \\
& \left.\mathbf{u}^{\varepsilon}\right|_{t=0}=0 \quad \text { on } \Omega^{\varepsilon}, \quad \int_{\Omega^{\varepsilon}} p^{\varepsilon} d x=0 .
\end{aligned}
$$

Let

$$
\mathcal{W}^{\varepsilon}=\left\{\varphi \in V\left(\Omega^{\varepsilon}\right)^{3}: \operatorname{div} \varphi=0 \text { in } \Omega^{\varepsilon}\right\},
$$

with the space $V\left(\Omega^{\varepsilon}\right)$ given by (5). From the theory of the Navier-Stokes equations, it is known that problem (7)-(11) admits a solution $\mathbf{u}^{\varepsilon} \in L^{2}\left(0, T ; \mathcal{W}^{\varepsilon}\right), \partial_{t} \mathbf{u}^{\varepsilon} \in$ $L^{4 / 3}\left(0, T ;\left(\mathcal{W}^{\varepsilon}\right)^{\prime}\right)$. Following [41] we determine the pressure field $p^{\varepsilon}$ from $\mathbf{u}^{\varepsilon}$. We refer to textbooks [31] and [42] for more details.

\subsection{The main result}

We start with the zeroth order approximation and consider the following problem on the fixed domain $\Omega \times(0, T)$ :

$$
\begin{aligned}
& \frac{\partial \mathbf{u}^{0}}{\partial t}+\operatorname{div}\left(\mathbf{u}^{\mathbf{0}} \otimes \mathbf{u}^{\mathbf{0}}\right)-\frac{1}{\mathbf{R e}} \operatorname{div}\left(2 D\left(\mathbf{u}^{\mathbf{0}}\right)-p^{0} I\right)=\mathbf{f} \text { in } Q_{T}, \\
& \operatorname{div} \mathbf{u}^{\mathbf{0}}=0 \quad \text { in } Q_{T}, \\
& \mathbf{u}^{0}=0 \quad \text { on }\left(\Sigma \cup \Sigma_{L}\right) \times(0, T), \\
& \mathbf{u}^{0} \quad \text { is periodic in }\left(x_{1}, x_{2}\right) \text { with period } L, \\
& \left.\mathbf{u}^{0}\right|_{t=0}=0 \quad \text { on } \Omega, \quad \int_{\Omega} p^{0} d x=0 .
\end{aligned}
$$

Using the function spaces

$$
V(\Omega)=\left\{\varphi \in H^{1}(\Omega): \varphi=0 \text { on } \Sigma_{L} \cup \Sigma, \varphi \text { is L-periodic in } x_{1}, x_{2}\right\}
$$


and

$$
\mathcal{W}=\left\{\varphi \in V(\Omega)^{3}: \operatorname{div} \varphi=0 \text { in } \Omega\right\},
$$

problem (13)-(17) admits a solution $\mathbf{u}^{0} \in L^{2}(0, T ; \mathcal{W})$, with $\partial_{t} \mathbf{u}^{0} \in L^{4 / 3}\left(0, T ;(\mathcal{W})^{\prime}\right)$, and, after extension by zero to $(0, L)^{2} \times(-\varepsilon, 0)$, it is also an element of $L^{2}\left(0, T ; \mathcal{W}^{\varepsilon}\right)$. Again we refer to [41] for determination of $p^{0}$.

Our aim is to prove that there are solutions to (7)-(11) close to $\mathbf{u}^{0}$. In this scope, we use a perturbation argument, which requires the following hypothesis on $\mathbf{u}^{0}$ :

(H1) Problem (13)-(17) admits a solution $\left(\mathbf{u}^{0}, p^{0}\right)$ such that $\nabla \mathbf{u}^{0} \in C^{2}\left(\bar{Q}_{T}\right)^{9}$, and $p^{0} \in C^{1}\left(\bar{Q}_{T}\right)$.

Obviously a solution satisfying hypothesis $(\mathbf{H 1})$ is unique. We notice that this hypothesis is always fulfilled in $2 \mathrm{D}$ and for stationary problems. Furthermore, we consider $p^{0}$ to be extended by its values on $\Sigma \times(0, T)$ to $(0, L)^{2} \times(-\varepsilon, 0) \times(0, T)$, i.e.

$$
p^{0}\left(x_{1}, x_{2}, x_{3}, t\right)=p^{0}\left(x_{1}, x_{2}, 0, t\right), \quad x_{3}<0, t \in(0, T) .
$$

Next, let the boundary layer functions $\left\{\beta^{j}, \omega^{j}\right\}, j=1,2$, be given by

$$
\begin{gathered}
-\triangle_{y} \beta^{j}+\nabla_{y} \omega^{j}=0 \quad \text { in } Z^{+} \cup\left(Y-\mathbf{e}^{3}\right) \\
\operatorname{div}_{y} \beta^{j}=0 \quad \text { in } Z_{b l} \\
{\left[\beta^{j}\right]_{S}(\cdot, 0)=0 \quad \text { on } S, \quad \omega^{j} \in L^{2}\left(Z_{b l}\right) \quad \text { and } \nabla_{y} \beta^{j} \in L^{2}\left(Z^{+} \cup\left(Y-\mathbf{e}^{3}\right)\right)^{3}} \\
{\left[\left\{\nabla_{y} \beta^{j}-\omega^{j} I\right\} \mathbf{e}^{3}\right]_{S}(\cdot, 0)=\left[\left\{2 D_{y}\left(\beta^{j}\right)-\omega^{j} I\right\} \mathbf{e}^{3}\right]_{S}(\cdot, 0)=\mathbf{e}^{j} \quad \text { on } S} \\
\beta^{j}=0 \text { on } \mathcal{B}, \quad\left\{\beta^{j}, \omega^{j}\right\} \text { is } y^{\prime}=\left(y_{1}, y_{2}\right)-\text { periodic }
\end{gathered}
$$

Here, $S=(0,1)^{2} \times\{0\}, Z^{+}=(0,1)^{2} \times(0,+\infty)$, and $Z_{b l}=Z^{+} \cup S \cup\left(Y-\mathbf{e}^{3}\right)$, see also Fig. 1. Furthermore let $M_{i j}=\frac{1}{|S|} \int_{S} \beta_{i}^{j} d y_{1} d y_{2}$ be Navier's matrix. The matrix $M$ is symmetric, and negatively definite. After [25], [28], [30] and [35] we have

$$
\left\{\begin{array}{l}
\left|D^{\alpha} \operatorname{curl}_{y} \beta^{j}(y)\right| \leq C e^{-2 \pi y_{3}}, \quad y_{3}>0, \alpha \in \mathbb{N}^{2} \cup(0,0) \\
\left|\beta^{j}(y)-\left(M_{1 j}, M_{2 j}, 0\right)\right| \leq C(\delta) e^{-\delta y_{3}}, y_{3}>0, \forall \delta<2 \pi \\
\left|D^{\alpha} \beta^{j}(y)\right| \leq C(\delta) e^{-\delta y_{3}}, y_{3}>0, \alpha \in \mathbb{N}^{2}, \forall \delta<2 \pi \\
\left|\omega^{j}(y)\right| \leq C e^{-2 \pi y_{3}}, \quad y_{3}>0 .
\end{array}\right.
$$

The effective problem is

$$
\begin{aligned}
& \frac{\partial \mathbf{u}^{e f f}}{\partial t}+\operatorname{div}\left(\mathbf{u}^{e f f} \otimes \mathbf{u}^{e f f}\right)-\frac{1}{\mathbf{R e}} \operatorname{div}\left(2 D\left(\mathbf{u}^{e f f}\right)-p^{e f f} I\right)=\mathbf{f} \text { in } Q_{T}, \\
& \operatorname{div} \mathbf{u}^{e f f}=0 \quad \text { in } Q_{T}, \\
& \mathbf{u}^{\text {eff }}=0 \quad \text { on } \Sigma_{L} \times(0, T), \quad \int_{\Omega} p^{e f f} d x=0, \\
& u_{3}^{e f f}=0, \quad \text { and } u_{k}^{e f f}=-\varepsilon \sum_{j=1}^{2} M_{k j} \frac{\partial}{\partial x_{3}} u_{j}^{e f f}, k=1,2, \quad \text { on } \Sigma \times(0, T) \text {, } \\
& \mathbf{u}^{\text {eff }} \text { is periodic in }\left(x_{1}, x_{2}\right) \text { with period } L \text {, } \\
& \left.\mathbf{u}^{e f f}\right|_{t=0}=0 \quad \text { on } \Omega .
\end{aligned}
$$

The main result of this article is the following error estimate 
Theorem 2. Let us suppose the hypothesis (H1) on the solution to the Navier-Stokes equations on the domain $\Omega$. Then we have

$$
\begin{aligned}
& \max _{0 \leq t \leq T}\left\|\nabla\left(\mathbf{u}^{\varepsilon}-\mathbf{u}^{e f f}\right)(t)\right\|_{L^{1}(\Omega)^{9}}^{2} \leq C \varepsilon^{2} \\
& \max _{0 \leq t \leq T}\left\|\left(\mathbf{u}^{\varepsilon}-\mathbf{u}^{e f f}\right)(t)\right\|_{L^{2}(\Omega)^{3}}^{2} \leq C \varepsilon^{3} \\
& \int_{0}^{T}\left\|\left(p^{\varepsilon}-p^{e f f}\right)(t)\right\|_{L_{l o c}^{2}(\Omega)^{3}}^{2} d t \leq C \varepsilon^{3} .
\end{aligned}
$$

\section{Zero order approximation}

Before stating the result on existence of solutions to (7)-(11) close to $\mathbf{u}^{0}$, we formulate the well know result about $L^{2}$-estimates for functions on thin domains.

Lemma 1. Let $\varphi \in V\left(\Omega^{\varepsilon}\right)$. Then we have

$$
\begin{aligned}
& \|\varphi\|_{L^{2}(\Sigma)} \leq C \varepsilon^{1 / 2}\left\|\nabla_{x} \varphi\right\|_{L^{2}\left(\Omega^{\varepsilon} \backslash \Omega\right)^{3}}, \\
& \|\varphi\|_{L^{2}\left(\Omega^{\varepsilon} \backslash \Omega\right)} \leq C \varepsilon\left\|\nabla_{x} \varphi\right\|_{L^{2}\left(\Omega^{\varepsilon} \backslash \Omega\right)^{3}} .
\end{aligned}
$$
$\mathbf{u}^{\varepsilon}$

Our first important result is the proof that $\mathbf{u}^{0}$ is a zeroth order approximation for

Proposition 1. Let us suppose hypothesis (H1), and let $\mathbf{f} \in C^{\infty}\left(\left([0, L]^{2} \times[-\varepsilon, L]\right) \times\right.$ $[0, T])^{3}$, supp $\mathbf{f} \subset\left([0, L]^{2} \times[-\varepsilon, L]\right) \times(0, T]$ be periodic in $\left(x_{1}, x_{2}\right)$ with period $L$. Then problem (7)-(11) has a solution $\left\{\mathbf{u}^{\varepsilon}, p^{\varepsilon}\right\} \in L^{2}\left(0, T ; \mathcal{W}^{\varepsilon} \cap H^{2}\left(\Omega^{\varepsilon}\right)^{3}\right) \times L^{2}\left(0, T ; H^{1}\left(\Omega^{\varepsilon}\right)\right)$, such that $\left\{\partial_{t} \mathbf{u}^{\varepsilon}, \partial_{t} p^{\varepsilon}\right\} \in L^{2}\left(0, T ; H^{1}\left(\Omega^{\varepsilon}\right)^{3}\right) \times L^{2}\left(0, T ; L^{2}\left(\Omega^{\varepsilon}\right)\right)$, and satisfying

$$
\begin{aligned}
& \int_{0}^{T}\left\|\nabla\left(\mathbf{u}^{\varepsilon}-\mathbf{u}^{0}\right)(t)\right\|_{L^{2}\left(\Omega^{\varepsilon}\right)^{9}}^{2} d t+\int_{0}^{T}\left\|\nabla \partial_{t}\left(\mathbf{u}^{\varepsilon}-\mathbf{u}^{0}\right)(t)\right\|_{L^{2}\left(\Omega^{\varepsilon}\right)^{9}}^{2} d t \leq C \varepsilon \\
& \max _{0 \leq t \leq T}\left(\left\|\left(\mathbf{u}^{\varepsilon}-\mathbf{u}^{0}\right)(t)\right\|_{L^{2}\left(\Omega^{\varepsilon}\right)^{3}}^{2}+\left\|\partial_{t}\left(\mathbf{u}^{\varepsilon}-\mathbf{u}^{0}\right)(t)\right\|_{L^{2}\left(\Omega^{\varepsilon}\right)^{3}}^{2}\right) \leq C \varepsilon .
\end{aligned}
$$

$\mathbf{u}^{\varepsilon}$ and $p^{\varepsilon}$ are uniquely determined.

Proof. We search for $\mathbf{u}^{\varepsilon}$ in the form $\mathbf{u}^{\varepsilon}=\mathbf{u}^{0}+\mathbf{w}^{\varepsilon}$. We are looking for $\mathbf{w}^{\varepsilon} \in L^{2}\left(0, T ; \mathcal{W}^{\varepsilon}\right)$, with $\partial_{t} \mathbf{w}^{\varepsilon} \in L^{2}\left(0, T ; \mathcal{W}^{\varepsilon}\right)$, and such that for all $\varphi \in \mathcal{W}^{\varepsilon}$, we have

$$
\begin{aligned}
& \int_{\Omega^{\varepsilon}}\left(\frac{\partial \mathbf{w}^{\varepsilon}}{\partial t}+\operatorname{div}\left(\mathbf{w}^{\varepsilon} \otimes \mathbf{w}^{\varepsilon}+\mathbf{w}^{\varepsilon} \otimes \mathbf{u}^{0}+\mathbf{u}^{0} \otimes \mathbf{w}^{\varepsilon}\right)\right) \varphi d x \\
& +\frac{1}{\operatorname{Re}} \int_{\Omega^{\varepsilon}} 2 D\left(\mathbf{w}^{\varepsilon}\right): D(\varphi) d x-\frac{1}{\operatorname{Re}} \int_{\Sigma}\left(2 D\left(\mathbf{u}^{0}\right)-p^{0} I\right) \mathbf{e}^{3} \varphi d S \\
& =\int_{\Omega^{\varepsilon} \backslash \Omega} \mathbf{f} \cdot \varphi d x, \\
& \mathbf{w}^{\varepsilon}(x, 0)=0 \text { on } \Omega^{\varepsilon} .
\end{aligned}
$$

The classical way of proving existence of a required smooth solution for (40)-(41) is to construct an approximate solution, to get uniform a priori estimates, and then to use the compactness method for passing to the limit. A problem related to (40)-(41) was studied in details in [26]. Here, we just prove the a priori estimates; the rest of the proof is identical to the corresponding result from [26]. Thus, we will manipulate $\mathbf{w}^{\varepsilon}$ as a smooth function. This can be justified by making manipulations with its Galerkin approximation or by working with the smooth solution defined for a short time. 
The proof of the a priori estimates will consist of several steps:

a) First, we insert $\mathbf{w}^{\varepsilon}$ as a test function in (40), and using estimates (36)-(37) from Lemma 1, the regularity properties of $\mathbf{f}$, and hypothesis $(\mathbf{H} \mathbf{1})$, we obtain for all $t \in(0, T)$

$$
\begin{aligned}
& \frac{1}{2} \frac{d}{d t} \int_{\Omega^{\varepsilon}}\left|\mathbf{w}^{\varepsilon}(t)\right|^{2} d x+\frac{2}{\operatorname{Re}} \int_{\Omega^{\varepsilon}}\left|D\left(\mathbf{w}^{\varepsilon}(t)\right)\right|^{2} d x+\int_{\Omega^{\varepsilon}}\left(\mathbf{w}^{\varepsilon}(t) \nabla\right) \mathbf{u}^{0} \mathbf{w}^{\varepsilon}(t) d x \\
& =\frac{1}{\operatorname{Re}} \int_{\Sigma}\left(2 D\left(\mathbf{u}^{0}\right)-p^{0} I\right)(t) \mathbf{e}^{3} \mathbf{w}^{\varepsilon}(t) d S+\int_{\Omega^{\varepsilon} \backslash \Omega} \mathbf{f}(t) \cdot \mathbf{w}^{\varepsilon}(t) d x \\
& \leq C\left\|\left(2 D\left(\mathbf{u}^{0}\right)-p^{0} I\right)(t)\right\|_{L^{2}(\Sigma)}\left\|\mathbf{w}^{\varepsilon}(t)\right\|_{L^{2}(\Sigma)}+C \varepsilon\left\|\nabla \mathbf{w}^{\varepsilon}(t)\right\|_{L^{2}\left(\Omega^{\varepsilon} \backslash \Omega\right)} \\
& \leq C \varepsilon^{1 / 2}\left\|\nabla \mathbf{w}^{\varepsilon}(t)\right\|_{L^{2}\left(\Omega^{\varepsilon} \backslash \Omega\right)} .
\end{aligned}
$$

Now, using again hypothesis (H1), which yields $\left\|\nabla \mathbf{u}^{0}\right\|_{L^{\infty}\left(Q_{T}\right)} \leq C$, we can estimate the triple term as follows

$$
\left|\int_{\Omega^{\varepsilon}}\left(\mathbf{w}^{\varepsilon}(t) \nabla\right) \mathbf{u}^{0}(t) \mathbf{w}^{\varepsilon}(t) d x d \tau\right| \leq C|| \nabla \mathbf{u}^{0} \|_{L^{\infty}\left(Q_{T}\right)} \int_{\Omega^{\varepsilon}}\left|\mathbf{w}^{\varepsilon}(t)\right|^{2} d x .
$$

Inserting the above estimate in (42), and using Gronwall's inequality yields

$$
\begin{aligned}
& \frac{1}{2} \frac{d}{d t} \int_{\Omega^{\varepsilon}}\left|\mathbf{w}^{\varepsilon}(t)\right|^{2} d x+\frac{2}{\operatorname{Re}} \int_{\Omega^{\varepsilon}}\left|D\left(\mathbf{w}^{\varepsilon}(t)\right)\right|^{2} d x \\
& \leq C \varepsilon^{1 / 2}\left\|\nabla \mathbf{w}^{\varepsilon}(t)\right\|_{L^{2}\left(\Omega^{\varepsilon} \backslash \Omega\right)}+C \varepsilon .
\end{aligned}
$$

After integrating in (43) with respect to t, and using Korn's inequality, we obtain the following estimates:

$$
\max _{0 \leq t \leq T}\left\|\mathbf{w}^{\varepsilon}(t)\right\|_{L^{2}\left(\Omega^{\varepsilon}\right)^{3}}^{2}+\int_{0}^{T}\left\|\nabla \mathbf{w}^{\varepsilon}(t)\right\|_{L^{2}\left(\Omega^{\varepsilon}\right)^{9}}^{2} d t \leq C \varepsilon .
$$

Next, evaluating weak formulation (40) at $t=0$ and taking into account $\mathbf{f}_{\{t=0\}}=0$, yields $\left.\partial_{t} \mathbf{u}^{0}\right|_{t=0}=0$ and $\left.p^{0}\right|_{t=0}=0$. Inserting as test function for (40) $\frac{\partial \mathbf{w}^{\varepsilon}}{\partial t}(0)$ gives

$$
\int_{\Omega^{\varepsilon}}\left|\frac{\partial \mathbf{w}^{\varepsilon}}{\partial t}(0)\right|^{2}=\frac{1}{\operatorname{Re}} \int_{\Sigma} p^{0}(0) I \mathbf{e}^{3} \frac{\partial \mathbf{w}^{\varepsilon}}{\partial t}(0) d S=0 .
$$

Thus we have $\frac{\partial \mathbf{w}^{\varepsilon}}{\partial t}(0)=0$ in $\Omega^{\varepsilon}$.

b) In order to prove the estimates for the time derivative, the following estimate will be useful. We reconsider equation (40) tested with $\mathbf{w}^{\varepsilon}$, and use estimate (44). We obtain the following estimate of $\left\|\nabla \mathbf{w}^{\varepsilon}(t)\right\|_{L^{2}\left(\Omega^{\varepsilon}\right)^{9}}^{2}$ for all $t$ from its smoothness interval

$$
\left\|\nabla \mathbf{w}^{\varepsilon}(t)\right\|_{L^{2}\left(\Omega^{\varepsilon}\right)^{9}}^{2} \leq c \sqrt{\varepsilon}\left(\sqrt{\varepsilon}+\left\|\partial_{t} \mathbf{w}^{\varepsilon}(t)\right\|_{L^{2}\left(\Omega^{\varepsilon}\right)^{3}}\right) .
$$

c) Now, we start to prove the estimates for the time derivative. To this end, we consider the variational problem for $\partial_{t} \mathbf{w}^{\varepsilon}$, which reads as follows:

$$
\begin{aligned}
& \int_{\Omega^{\varepsilon}} \frac{\partial\left(\partial_{t} \mathbf{w}^{\varepsilon}\right)}{\partial t} \varphi+\int_{\Omega^{\varepsilon}} \operatorname{div}\left(\partial_{t} \mathbf{w}^{\varepsilon} \otimes \mathbf{w}^{\varepsilon}+\mathbf{w}^{\varepsilon} \otimes \partial_{t} \mathbf{w}^{\varepsilon}\right) \varphi d x \\
& +\int_{\Omega^{\varepsilon}} \operatorname{div}\left(\partial_{t} \mathbf{w}^{\varepsilon} \otimes \mathbf{u}^{0}+\mathbf{w}^{\varepsilon} \otimes \partial_{t} \mathbf{u}^{0}+\partial_{t} \mathbf{u}^{0} \otimes \mathbf{w}^{\varepsilon}+\mathbf{u}^{0} \otimes \partial_{t} \mathbf{w}^{\varepsilon}\right) \varphi d x \\
& +\frac{1}{\mathbf{R e}} \int_{\Omega^{\varepsilon}} 2 D\left(\partial_{t} \mathbf{w}^{\varepsilon}\right): D(\varphi) d x-\frac{1}{\mathbf{R e}} \int_{\Sigma}\left(2 D\left(\partial_{t} \mathbf{u}^{0}\right)-\partial_{t} p^{0} I\right) \mathbf{e}^{3} \varphi d S
\end{aligned}
$$




$$
\begin{aligned}
& =\int_{\Omega^{\varepsilon} \backslash \Omega} \partial_{t} \mathbf{f} \cdot \varphi d x, \quad \forall \varphi \in \mathcal{W}^{\varepsilon}, \\
& \partial_{t} \mathbf{w}^{\varepsilon}(x, 0)=0 \quad \text { on } \Omega^{\varepsilon} .
\end{aligned}
$$

We test (47) with $\partial_{t} \mathbf{w}^{\varepsilon}$ and estimate the terms. In difference with the system (40)(41), when testing the problem (47)-(48) by $\partial_{t} \mathbf{w}^{\varepsilon}$, the time derivative of the inertia term gives a contribution. Using estimate (46), the corresponding terms are estimated as follows. First, we have

$$
\begin{aligned}
& \left|\int_{\Omega^{\varepsilon}}\left(\partial_{t} \mathbf{w}^{\varepsilon} \nabla\right) \mathbf{w}^{\varepsilon} \partial_{t} \mathbf{w}^{\varepsilon}\right| \leq c\left\|\nabla \mathbf{w}^{\varepsilon}(t)\right\|_{L^{2}\left(\Omega^{\varepsilon}\right)^{9}}\left\|\nabla \partial_{t} \mathbf{w}^{\varepsilon}(t)\right\|_{L^{2}\left(\Omega^{\varepsilon}\right)^{9}}^{2} \\
& \leq c \varepsilon^{1 / 4}\left(\varepsilon^{1 / 4}+\left\|\partial_{t} \mathbf{w}^{\varepsilon}(t)\right\|_{L^{2}\left(\Omega^{\varepsilon}\right)^{3}}^{1 / 2}\left\|\nabla \partial_{t} \mathbf{w}^{\varepsilon}(t)\right\|_{L^{2}\left(\Omega^{\varepsilon}\right)^{9} .}^{2} .\right.
\end{aligned}
$$

The "Oseen like" terms behave slightly better. A typical estimate reads

$$
\begin{aligned}
& \left|\int_{\Omega^{\varepsilon}}\left(\partial_{t} \mathbf{u}^{0} \nabla\right) \mathbf{w}^{\varepsilon} \partial_{t} \mathbf{w}^{\varepsilon}\right| \\
& \leq c\left\|\nabla \mathbf{w}^{\varepsilon}(t)\right\|_{L^{2}\left(\Omega^{\varepsilon}\right)^{9}}\left\|\nabla \partial_{t} \mathbf{w}^{\varepsilon}(t)\right\|_{L^{2}\left(\Omega^{\varepsilon}\right)^{9}}\left\|\nabla \partial_{t} \mathbf{u}^{0}(t)\right\|_{L^{2}\left(\Omega^{\varepsilon}\right)^{9}} \\
& \leq c \varepsilon^{1 / 4}\left(\varepsilon^{1 / 4}+\left\|\partial_{t} \mathbf{w}^{\varepsilon}(t)\right\|_{L^{2}\left(\Omega^{\varepsilon}\right)^{3}}^{1 / 2}\left\|\nabla \partial_{t} \mathbf{w}^{\varepsilon}(t)\right\|_{L^{2}\left(\Omega^{\varepsilon}\right)^{9}}\left\|\nabla \partial_{t} \mathbf{u}^{0}(t)\right\|_{L^{2}\left(\Omega^{\varepsilon}\right)^{9}}\right.
\end{aligned}
$$

After inserting (49)-(50) into equation (47) tested by $\partial_{t} \mathbf{w}^{\varepsilon}$, and using Lemma 1 , we obtain the following differential inequality

$$
\frac{1}{2} \frac{d}{d t} \int_{\Omega^{\varepsilon}}\left|\partial_{t} \mathbf{w}^{\varepsilon}(t)\right|^{2}+\left\|\nabla \partial_{t} \mathbf{w}^{\varepsilon}(t)\right\|_{L^{2}\left(\Omega^{\varepsilon}\right)^{9}}^{2}\left\{1-c \varepsilon^{1 / 4}\left\|\partial_{t} \mathbf{w}^{\varepsilon}(t)\right\|_{L^{2}\left(\Omega^{\varepsilon}\right)^{9}}^{1 / 2} \leq C \varepsilon .\right.
$$

Now for all $t$ from the smoothness interval and such that $\left\|\partial_{t} \mathbf{w}^{\varepsilon}(t)\right\|_{L^{2}\left(\Omega^{\varepsilon}\right)^{9}} \leq C \varepsilon^{-1 / 2}$, we have

$$
\left\|\partial_{t} \mathbf{w}^{\varepsilon}(t)\right\|_{L^{2}\left(\Omega^{\varepsilon}\right)^{9}}^{2} \leq C \varepsilon .
$$

Therefore the estimate (52) remains valid at the smoothness interval.

d) Finally, we see that the estimates (38)-(39) remain valid on $(0, T)$. These a priori estimates allow proving existence on standard way. By the obtained regularity, uniqueness is obvious.

Proposition 2. For the solution to (7)-(11), constructed in Proposition 1, the following estimates hold:

$$
\begin{aligned}
& \left\|\mathbf{u}^{\varepsilon}\right\|_{L^{2}\left(\Omega^{\varepsilon} \backslash \Omega \times(0, T)\right)^{3}}+\left\|\partial_{t} \mathbf{u}^{\varepsilon}\right\|_{L^{2}\left(\Omega^{\varepsilon} \backslash \Omega \times(0, T)\right)^{3}} \leq C \varepsilon \sqrt{\varepsilon} \\
& \left\|\mathbf{u}^{\varepsilon}\right\|_{L^{2}(\Sigma \times(0, T))^{3}}+\left\|\partial_{t} \mathbf{u}^{\varepsilon}\right\|_{L^{2}(\Sigma \times(0, T))^{3}} \leq C \varepsilon \\
& \left\|\mathbf{u}^{\varepsilon}-\mathbf{u}^{0}\right\|_{L^{2}\left(Q_{T}\right)^{3}}+\left\|\partial_{t}\left(\mathbf{u}^{\varepsilon}-\mathbf{u}^{0}\right)\right\|_{L^{2}\left(Q_{T}\right)^{3}} \leq C \varepsilon \\
& \left\|p^{\varepsilon}-p^{0}\right\|_{L_{0}^{2}\left(Q_{T}\right)} \leq C \sqrt{\varepsilon} .
\end{aligned}
$$

Proof. Estimates (53) and (54) are direct consequences of Poincaré's inequality (36) and the trace inequality (37) in $\Omega^{\varepsilon} \backslash \Omega$, respectively.

In order to get the estimate (55), we note that $\mathbf{w}^{\varepsilon}=\mathbf{u}^{\varepsilon}-\mathbf{u}^{0}$ and $\pi^{\varepsilon}=p^{\varepsilon}-p^{0}$ satisfy the system

$$
\begin{gathered}
\frac{\partial \mathbf{w}^{\varepsilon}}{\partial t}+\operatorname{div}\left(\mathbf{w}^{\varepsilon} \otimes \mathbf{u}^{0}+\mathbf{u}^{0} \otimes \mathbf{w}^{\varepsilon}\right)-\frac{1}{\mathbf{R e}} \operatorname{div}\left(2 D\left(\mathbf{w}^{\varepsilon}\right)-\pi^{\varepsilon} I\right)= \\
-\operatorname{div}\left(\mathbf{w}^{\varepsilon} \otimes \mathbf{w}^{\varepsilon}\right) \text { in } Q_{T} ;
\end{gathered}
$$




$$
\begin{gathered}
\operatorname{div} \mathbf{w}^{\varepsilon}=0 \quad \text { in } Q_{T} ; \quad \mathbf{w}^{\varepsilon}(x, 0)=0 \quad \text { in } \Omega ; \\
\mathbf{w}^{\varepsilon}=\xi \text { on } \quad \Sigma_{T}^{U}=\left(\Sigma \cup \Sigma_{L}\right) \times(0, T), \quad \int_{\Sigma \cup \Sigma_{L}} \xi \cdot \nu d S=0 ; \\
\left\{\mathbf{w}^{\varepsilon}, \pi^{\varepsilon}\right\}, \text { is L-periodic in }\left(x_{1}, x_{2}\right)
\end{gathered}
$$

By the hypothesis (H1) $\nabla \mathbf{u}^{0} \in L^{\infty}\left(Q_{T}\right)^{9}$ and by (54), we have $\|\xi\|_{L^{2}\left(\Sigma_{T}^{U}\right)^{3}} \leq C \varepsilon$. Next, we have $\left\|\mathbf{w}^{\varepsilon} \otimes \mathbf{w}^{\varepsilon}\right\|_{L^{2}\left(Q_{T}\right)^{9}} \leq C \varepsilon$. Now, we apply the theory of the very weak solutions for the Oseen system developed in Section 6, Proposition 8 and conclude that the inequality (55) holds true.

The estimate (56) follows from the first equation in (57) and Nečas' inequality in $\Omega$. This proves the proposition.

\section{First order approximation}

In the above considerations, we have obtained the uniform a priori estimates for $\left\{\mathbf{u}^{\varepsilon}, p^{\varepsilon}\right\}$. Moreover, we have found that the flow equations with truncated asperities (13)-(17), posed in $\Omega$, give an $O(\varepsilon) L^{2}$-approximation for $\mathbf{u}^{\varepsilon}$. At $\Sigma$ it is an $O(\varepsilon)$ $L^{2}$-approximation.

Following the approach from [28], the Navier slip condition should correspond to taking into the account the next order corrections for the velocity. Then formally we set

$$
\begin{aligned}
\mathbf{u}^{\varepsilon}=\mathbf{u}^{0} & -\left.\varepsilon \sum_{j=1}^{2}\left(\beta^{j}\left(\frac{x}{\varepsilon}\right)-\left(M_{j 1}, M_{j 2}, 0\right) H\left(x_{3}\right)\right) \frac{\partial u_{j}^{0}}{\partial x_{3}}\right|_{\Sigma} \\
& -\varepsilon H\left(x_{3}\right) \sum_{j, k=1}^{2} M_{k j} \mathbf{g}^{k j}(x, t)+O\left(\varepsilon^{2}\right)
\end{aligned}
$$

where the boundary layer functions $\beta^{j}$ are given by (21)-(25) and the last term corresponds to the counterflow generated by stabilization of the boundary layer functions $\beta^{j}$. It is given through the systems

$$
\left\{\begin{array}{l}
\frac{\partial \mathbf{g}^{k j}}{\partial t}+\operatorname{div}\left(\mathbf{g}^{k j} \otimes \mathbf{u}^{0}+\mathbf{u}^{0} \otimes \mathbf{g}^{k j}\right) \\
-\frac{1}{\mathbf{R e}} \operatorname{div}\left(2 D\left(\mathbf{g}^{k j}\right)-p_{g}^{k j} I\right)=0 \text { in } Q_{T} ; \\
\operatorname{div} \mathbf{g}^{k j}=0 \quad \text { in } Q_{T} ; \\
\mathbf{g}^{k j}(x, 0)=0 \quad \text { in } \Omega ; \\
\mathbf{g}^{k j}=\frac{\partial u_{j}^{0}}{\partial x_{3}} \mathbf{e}^{k} \text { on } \Sigma \times(0, T), \quad \mathbf{g}^{k j}=0 \text { on } \Sigma_{L} \times(0, T) ; \\
\left\{\mathbf{g}^{k j}, p_{g}^{k j}\right\}, \text { is L-periodic in }\left(x_{1}, x_{2}\right)
\end{array}\right.
$$

On the interface $\Sigma$, we have

$$
\frac{\partial u_{i}}{\partial x_{3}}=\frac{\partial u_{i}^{0}}{\partial x_{3}}-\sum_{j=1}^{2} \frac{\partial \beta_{i}^{j}}{\partial y_{3}}\left(\frac{x}{\varepsilon}\right) \frac{\partial u_{j}^{0}}{\partial x_{3}}+O(\varepsilon) \quad \text { and } \quad \frac{1}{\varepsilon} u_{i}=-\sum_{j=1}^{2} \beta_{i}^{j}\left(\frac{x}{\varepsilon}\right) \frac{\partial u_{j}^{0}}{\partial x_{3}}+O(\varepsilon) .
$$


After averaging we obtain the familiar form of the Navier's slip condition

$$
u_{i}^{e f f}=-\varepsilon \sum_{j=1}^{2} M_{j i} \frac{\partial u_{j}^{e f f}}{\partial x_{3}} \quad \text { on } \Sigma,
$$

where $u^{e f f}$ is the average over the impurities. The higher order terms are neglected.

Now, let us make this formal asymptotic expansion rigorous. Let

$$
\beta^{j, \varepsilon}(x)=\varepsilon \beta^{j}(x / \varepsilon) \quad \text { and } \quad \omega^{j, \varepsilon}(x)=\omega^{j}(x / \varepsilon), \quad j=1,2 .
$$

We introduce the velocity and pressure error functions by

$$
\begin{aligned}
\mathcal{U}_{0}^{\varepsilon}(x, t) & =\mathbf{u}^{\varepsilon}-\mathbf{u}^{0}+\left.H\left(x_{3}\right) \sum_{j=1}^{2}\left(\beta^{j, \varepsilon}(x)-\varepsilon\left(M_{1 j}, M_{2 j}, 0\right)\right) \frac{\partial u_{j}^{0}}{\partial x_{3}}\right|_{\Sigma} \\
& +\varepsilon H\left(x_{3}\right) \sum_{j, k=1}^{2} M_{k j} \mathbf{g}^{k j}(x, t)+\left.\sum_{j=1}^{2} \beta^{j, \varepsilon}(x) H\left(-x_{3}\right) \frac{\partial u_{j}^{0}}{\partial x_{3}}\right|_{\Sigma} \\
\mathcal{P}_{0}^{\varepsilon}(x, t) & =p^{\varepsilon}-p^{0}+\left.\sum_{j=1}^{2} \omega^{j, \varepsilon} \frac{\partial u_{j}^{0}}{\partial x_{3}}\right|_{\Sigma}+\varepsilon H\left(x_{3}\right) \sum_{j, k=1}^{2} M_{k j} p_{g}^{k j} .
\end{aligned}
$$

Also the abbreviation $r_{j}=\left.\frac{\partial u_{j}^{0}}{\partial x_{3}}\right|_{\Sigma}$ will be used. We rewrite (40)-(41) in the form

$$
\begin{aligned}
& \int_{\Omega^{\varepsilon}}\left(\frac{\partial}{\partial t} \mathbf{w}^{\varepsilon}+\operatorname{div}\left(\mathbf{w}^{\varepsilon} \otimes \mathbf{u}^{0}+\mathbf{u}^{0} \otimes \mathbf{w}^{\varepsilon}\right)\right) \varphi d x \\
& +\frac{1}{\operatorname{Re}} \int_{\Omega^{\varepsilon}}\left(2 D\left(\mathbf{w}^{\varepsilon}\right)-\left(p^{\varepsilon}-p^{0}\right) I\right): D(\varphi) d x-\frac{1}{\operatorname{Re}} \int_{\Sigma} \sum_{j=1}^{2} r_{j} \varphi_{j} d S \\
& =\int_{\Omega^{\varepsilon} \backslash \Omega}\left(\mathbf{f}-\nabla p^{0}\right) \cdot \varphi d x-\int_{\Omega^{\varepsilon}} \operatorname{div}\left(\mathbf{w}^{\varepsilon} \otimes \mathbf{w}^{\varepsilon}\right) \varphi d x, \forall \varphi \in V\left(\Omega^{\varepsilon}\right), \\
& \mathbf{w}^{\varepsilon}(x, 0)=0 \quad \text { on } \Omega^{\varepsilon} .
\end{aligned}
$$

Then, if we denote by $\mathbf{M}^{\mathbf{j}}=\left(\mathbf{M}_{\mathbf{1} \mathbf{j}}, \mathbf{M}_{\mathbf{2} \mathbf{j}}, \mathbf{0}\right)$ for $j=1,2$, we have

$$
\begin{aligned}
& \int_{\Omega^{\varepsilon}} \frac{\partial}{\partial t}\left(\left(\beta^{j, \varepsilon}-\varepsilon H\left(x_{3}\right) \mathbf{M}^{\mathbf{j}}\right) \mathrm{r}_{\mathbf{j}}\right) \varphi \mathrm{dx} \\
& +\int_{\Omega^{\varepsilon}} \operatorname{div}\left(\left(\beta^{j, \varepsilon}-\varepsilon H\left(x_{3}\right) \mathbf{M}^{\mathbf{j}}\right) \mathrm{r}_{\mathbf{j}} \otimes \mathbf{u}^{0}+\mathbf{u}^{0} \otimes\left(\beta^{\mathrm{j}, \varepsilon}-\varepsilon \mathrm{H}\left(\mathrm{x}_{3}\right) \mathbf{M}^{\mathbf{j}}\right) \mathrm{r}_{\mathrm{j}}\right) \varphi \mathrm{dx} \\
& +\frac{1}{\operatorname{Re}} \int_{\Omega^{\varepsilon}}\left(2 D\left(\left(\beta^{j, \varepsilon}-\varepsilon H\left(x_{3}\right) \mathbf{M}^{\mathbf{j}}\right) \mathrm{r}_{\mathrm{j}}\right)-\omega^{\mathrm{j}, \varepsilon} \mathrm{r}_{\mathrm{j}} \mathrm{I}\right): \mathrm{D}(\varphi) \mathrm{dx}+\frac{1}{\boldsymbol{R e}} \int_{\Sigma} \mathrm{r}_{\mathrm{j}} \varphi_{\mathrm{j}} \mathrm{dS} \\
& =\frac{1}{\mathbf{R e}} \int_{\Omega^{\varepsilon}} 2 \operatorname{sym}\left(\left(\beta^{j, \varepsilon}-\varepsilon \mathbf{M}^{\mathbf{j}} \mathrm{H}\left(\mathrm{x}_{3}\right)\right) \otimes \nabla \mathrm{r}_{\mathrm{j}}\right): \mathrm{D}(\varphi) \mathrm{dx} \\
& +\frac{1}{\boldsymbol{R e}} \int_{\Omega^{\varepsilon}}\left(\beta^{j, \varepsilon}-\varepsilon \mathbf{M}^{\mathbf{j}} \mathrm{H}\left(\mathrm{x}_{3}\right)\right) \cdot \sum_{\mathrm{k}=1}^{2} \frac{\partial}{\partial \mathrm{x}_{\mathrm{k}}}\left(\frac{\partial \mathrm{r}_{\mathrm{j}}}{\partial \mathrm{x}_{\mathrm{k}}} \varphi\right) \mathrm{d} \mathrm{x} \\
& +\frac{1}{\operatorname{Re}} \int_{\Omega^{\varepsilon}}\left(\beta^{j, \varepsilon}-\varepsilon \mathbf{M}^{\mathbf{j}} \mathrm{H}\left(\mathrm{x}_{3}\right)\right) \cdot \sum_{\mathrm{i}=1}^{3} \frac{\partial}{\partial \mathrm{x}_{\mathrm{i}}}\left(\varphi_{\mathrm{i}} \nabla \mathrm{r}_{\mathrm{j}}\right) \mathrm{dx} \\
& +\frac{\varepsilon}{\boldsymbol{R e}} \int_{\Sigma} \varphi_{3} \mathbf{M}^{\mathbf{j}} \cdot \nabla \mathrm{r}_{\mathrm{j}} \mathrm{dS}+\frac{1}{\boldsymbol{R e}} \int_{\Omega^{\varepsilon}} \omega^{\mathrm{j}, \varepsilon} \varphi \cdot \nabla \mathrm{r}_{\mathrm{j}} \mathrm{dx}+\int_{\Omega^{\varepsilon}} \frac{\partial \mathrm{r}_{\mathrm{j}}}{\partial \mathrm{t}}\left(\beta^{\mathrm{j}, \varepsilon}-\varepsilon \mathrm{H}\left(\mathrm{x}_{3}\right) \mathbf{M}^{\mathbf{j}}\right) \varphi \mathrm{dx} \\
& +\int_{\Omega^{\varepsilon}} \operatorname{div}\left(\left(\beta^{j, \varepsilon}-\varepsilon \mathbf{M}^{\mathbf{j}}\right) \mathrm{r}_{\mathbf{j}} \otimes \mathbf{u}^{0}+\mathbf{u}^{0} \otimes\left(\beta^{\mathrm{j}, \varepsilon}-\varepsilon \mathbf{M}^{\mathbf{j}}\right) \mathrm{r}_{\mathrm{j}}\right) \varphi \mathrm{dx} \\
& =<\Phi^{1, j}(t), \varphi>_{V^{\prime}\left(\Omega^{\varepsilon}\right), V\left(\Omega^{\varepsilon}\right)} \text {. }
\end{aligned}
$$


Lemma 2. We have

$$
\int_{0}^{T}\left|<\Phi^{1, j}(t), \varphi>_{V^{\prime}\left(\Omega^{\varepsilon}\right), V\left(\Omega^{\varepsilon}\right)}\right| d t \leq C \varepsilon^{3 / 2}\|\nabla \varphi\|_{L^{2}\left(\Omega^{\varepsilon} \times(0, T)\right)} .
$$

Proof. The estimate follows from the decay of the boundary layer functions and Lemma 1. We note that the mean of $\omega^{j}$ over sections in $Z^{+}$is zero and its mean over $Z^{+}$ is zero. Then the term $\int_{\Omega} \omega^{j, \varepsilon} \varphi \cdot \nabla r_{j} d x$ is estimated the functions $\delta^{j}$, such that $\operatorname{div}_{y} \delta^{j}=\omega^{j}$ in $Z^{+}, \delta_{3}^{j}=0$ on $S$ and $\delta^{j}$ is 1-periodic in $y_{1}, y_{2}$. Estimating other terms is straightforward.

Remark 1. Functions $\mathbf{g}^{k j}$ are added in (63) in order to have the trace continuity on $\Sigma$ and $\mathcal{U}_{0}^{\varepsilon} \in H^{1}$.

We summarize the obtained equations and estimates:

Proposition 3. Let $\left\{\mathcal{U}_{0}^{\varepsilon}, \mathcal{P}_{0}^{\varepsilon}\right\}$ be given by (63)-(64). Then we have $\mathcal{U}_{0}^{\varepsilon} \in H^{1}\left(\Omega^{\varepsilon} \times\right.$ $(0, T))$ and $\left\{\mathcal{U}_{0}^{\varepsilon}, \mathcal{P}_{0}^{\varepsilon}\right\}$ satisfies the variational equation

$$
\begin{aligned}
& \int_{\Omega^{\varepsilon}}\left(\frac{\partial \mathcal{U}_{0}^{\varepsilon}}{\partial t}+\operatorname{div}\left(\mathcal{U}_{0}^{\varepsilon} \otimes \mathbf{u}^{0}+\mathbf{u}^{0} \otimes \mathcal{U}_{0}^{\varepsilon}\right)\right) \varphi d x+\frac{1}{\mathbf{R e}} \int_{\Omega^{\varepsilon}}\left(2 D\left(\mathcal{U}_{0}^{\varepsilon}\right)-\mathcal{P}_{0}^{\varepsilon} I\right): D(\varphi) d x \\
& \quad=\int_{\Omega^{\varepsilon} \backslash \Omega}\left(\mathbf{f}-\nabla p^{0}\right) \cdot \varphi d x-\int_{\Omega^{\varepsilon}} \operatorname{div}\left(\left(\mathcal{U}_{0}^{\varepsilon}-\mathbf{w}^{\varepsilon}\right) \otimes \mathbf{w}^{\varepsilon}\right) \varphi d x \\
& -\sum_{j=1}^{2}<\Phi^{1, j}(t), \varphi>_{V^{\prime}\left(\Omega^{\varepsilon}\right), V\left(\Omega^{\varepsilon}\right)}=<\Phi^{1}(t), \varphi>_{V^{\prime}\left(\Omega^{\varepsilon}\right), V\left(\Omega^{\varepsilon}\right)} \\
& \quad \mathcal{U}_{0}^{\varepsilon}(x, 0)=0 \quad \text { on } \Omega^{\varepsilon}
\end{aligned}
$$

Finally, we have

$$
\operatorname{div} \mathcal{U}_{0}^{\varepsilon}=-\sum_{j=1}^{2}\left(\left(\beta^{j, \varepsilon}-\varepsilon \mathbf{M}^{\mathbf{j}} \mathrm{H}\left(\mathrm{x}_{3}\right)\right) \cdot \nabla \mathrm{r}_{\mathrm{j}} .\right.
$$

Proposition 4. We have

$$
\begin{gathered}
\int_{0}^{T}\left|<\Phi^{1}(t), \varphi>_{V^{\prime}\left(\Omega^{\varepsilon}\right), V\left(\Omega^{\varepsilon}\right)}\right| d t \leq C \varepsilon^{3 / 2}\|\nabla \varphi\|_{L^{2}\left(\Omega^{\varepsilon} \times(0, T)\right)}, \\
\left\|\operatorname{div} \mathcal{U}_{0}^{\varepsilon}\right\|_{L^{2}\left(\Omega^{\varepsilon} \times(0, T)\right)} \leq C \varepsilon^{3 / 2} .
\end{gathered}
$$

Proof. In fact it is enough to comment on the term $\int_{\Omega^{\varepsilon}} \operatorname{div}\left(\left(\mathcal{U}_{0}^{\varepsilon}-\mathbf{w}^{\varepsilon}\right) \otimes \mathbf{w}^{\varepsilon}\right) \varphi d x$. Let us mention that part of this term we got by simple manipulation of type $\int_{\Omega^{\varepsilon}} \operatorname{div}\left(\mathcal{U}_{0}^{\varepsilon} \otimes\right.$ $\left.\mathbf{u}^{0}\right) \varphi d x=\int_{\Omega^{\varepsilon}} \operatorname{div}\left(\mathcal{U}_{0}^{\varepsilon} \otimes \mathbf{u}^{\varepsilon}\right) \varphi d x-\int_{\Omega^{\varepsilon}} \operatorname{div}\left(\left(\mathcal{U}_{0}^{\varepsilon} \otimes \mathbf{w}^{\varepsilon}\right) \varphi d x\right)$. Moreover we observe that || $\mathcal{U}_{0}^{\varepsilon}-\left.\mathbf{w}^{\varepsilon}\right|_{L^{\infty}\left(Q_{T}\right)} \leq C \varepsilon$ and the estimate (72) follows immediately.

Elimination of the pressure field requires solenoidal test functions. As consequence of Theorem 1 we conclude that there is $\mathbf{z}^{\varepsilon} \in H^{1}\left(\Omega^{\varepsilon} \backslash\left\{x_{3} \geq \varepsilon\right\}\right)$, div $\mathbf{z}^{\varepsilon}=\operatorname{div} \mathcal{U}_{0}^{\varepsilon}$, such that $\mathbf{z}^{\varepsilon}=0$ on $\mathcal{B}^{\varepsilon} \times(0, T)$ and on $\left\{x_{3}=\varepsilon\right\} \times(0, T)$ and $\mathbf{z}^{\varepsilon}$ is $L$-periodic $x_{1}, x_{2}$. Finally

$$
\left\|\mathbf{z}^{\varepsilon}\right\|_{L^{2}\left(0, T ; H^{1}\left(\Omega^{\varepsilon} \backslash\left\{x_{3} \geq \varepsilon\right\}\right)\right)}+\left\|\partial_{t} \mathbf{z}^{\varepsilon}\right\|_{L^{2}\left(0, T ; H^{1}\left(\Omega^{\varepsilon} \backslash\left\{x_{3} \geq \varepsilon\right\}\right)\right)} \leq C \varepsilon^{3 / 2} .
$$


It remains to write the problem for $\mathcal{U}_{0}^{\varepsilon}-\mathbf{z}^{\varepsilon}$ :

$$
\begin{gathered}
\int_{\Omega^{\varepsilon}}\left(\frac{\partial\left(\mathcal{U}_{0}^{\varepsilon}-\mathbf{z}^{\varepsilon}\right)}{\partial t}+\operatorname{div}\left(\left(\mathcal{U}_{0}^{\varepsilon}-\mathbf{z}^{\varepsilon}\right) \otimes \mathbf{u}^{0}+\mathbf{u}^{0} \otimes\left(\mathcal{U}_{0}^{\varepsilon}-\mathbf{z}^{\varepsilon}\right)\right)\right) \varphi d x+ \\
\frac{1}{\operatorname{Re}} \int_{\Omega^{\varepsilon}} 2 D\left(\mathcal{U}_{0}^{\varepsilon}-\mathbf{z}^{\varepsilon}\right): D(\varphi) d x=<\Psi(t), \varphi>_{V^{\prime}\left(\Omega^{\varepsilon}\right), V\left(\Omega^{\varepsilon}\right)}, \\
\left(\mathcal{U}_{0}^{\varepsilon}-\mathbf{z}^{\varepsilon}\right)(x, 0)=0 \quad \text { on } \Omega^{\varepsilon},
\end{gathered}
$$

where

$$
\int_{0}^{T}\left|<\Psi(t), \varphi>_{V^{\prime}\left(\Omega^{\varepsilon}\right), V\left(\Omega^{\varepsilon}\right)}\right| d t \leq C \varepsilon^{3 / 2}\|\nabla \varphi\|_{L^{2}\left(\Omega^{\varepsilon} \times(0, T)\right)} .
$$

Then we have

Proposition 5. Let us suppose the hypothesis (H1) and let $\mathbf{f} \in C^{\infty}\left(\overline{\Omega^{\varepsilon}} \times[0, T]\right)^{3}$, be such that supp $\mathbf{f} \subset \overline{\Omega^{\varepsilon}} \times(0, T]$, and it is periodic in $\left(x_{1}, x_{2}\right)$ with period $L$. Then we have

$$
\begin{gathered}
\int_{0}^{T}\left(\left\|\nabla\left(\mathcal{U}_{0}^{\varepsilon}(t)-\mathbf{z}^{\varepsilon}\right)\right\|_{L^{2}\left(\Omega^{\varepsilon}\right)^{9}}^{2}+\left\|\nabla \partial_{t}\left(\mathcal{U}_{0}^{\varepsilon}(t)-\mathbf{z}^{\varepsilon}(t)\right)\right\|_{L^{2}\left(\Omega^{\varepsilon}\right)^{9}}^{2}\right) d t \leq C \varepsilon^{3} \\
\max _{0 \leq t \leq T}\left(\left\|\left(\mathcal{U}_{0}^{\varepsilon}(t)-\mathbf{z}^{\varepsilon}(t)\right)\right\|_{L^{2}\left(\Omega^{\varepsilon}\right)^{3}}^{2}+\left\|\partial_{t}\left(\mathcal{U}_{0}^{\varepsilon}(t)-\mathbf{z}^{\varepsilon}(t)\right)\right\|_{L^{2}\left(\Omega^{\varepsilon}\right)^{3}}^{2}\right) \leq C \varepsilon^{3} \\
\left\|\mathcal{U}_{0}^{\varepsilon}-\mathbf{z}^{\varepsilon}\right\|_{L^{2}\left(\Omega^{\varepsilon} \backslash \Omega \times(0, T)\right)^{3}}+\left\|\partial_{t}\left(\mathcal{U}_{0}^{\varepsilon}-\mathbf{z}^{\varepsilon}\right)\right\|_{L^{2}\left(\Omega^{\varepsilon} \backslash \Omega \times(0, T)\right)^{3}} \leq C \varepsilon^{5 / 2} \\
\left\|\mathcal{U}_{0}^{\varepsilon}-\mathbf{z}^{\varepsilon}\right\|_{L^{2}(\Sigma \times(0, T))^{3}}+\left\|\partial_{t}\left(\mathcal{U}_{0}^{\varepsilon}-\mathbf{z}^{\varepsilon}\right)\right\|_{L^{2}(\Sigma \times(0, T))^{3}} \leq C \varepsilon^{2} \\
\left\|\mathcal{U}_{0}^{\varepsilon}-\mathbf{z}^{\varepsilon}\right\|_{L^{2}\left(Q_{T}\right)^{3}}+\left\|\partial_{t}\left(\mathcal{U}_{0}^{\varepsilon}-\mathbf{z}^{\varepsilon}\right)\right\|_{L^{2}\left(Q_{T}\right)^{3}} \leq C \varepsilon^{2} \\
\left\|\mathcal{P}^{\varepsilon}\right\|_{L_{0}^{2}\left(Q_{T}\right)} \leq C \varepsilon^{3 / 2} .
\end{gathered}
$$

Proof. We take $\varphi=\mathcal{U}_{0}^{\varepsilon}-\mathbf{z}^{\varepsilon}$ as test function. Then after repeating the calculations from the proof of Proposition 1 a), we get

$$
\max _{0 \leq t \leq T}\left\|\mathcal{U}_{0}^{\varepsilon}-\mathbf{z}^{\varepsilon}\right\|_{L^{2}\left(\Omega^{\varepsilon}\right)^{3}}^{2}+\int_{0}^{T}\left\|\nabla\left(\mathcal{U}_{0}^{\varepsilon}-\mathbf{z}^{\varepsilon}\right)\right\|_{L^{2}\left(\Omega^{\varepsilon}\right)^{9}}^{2} d t \leq C \varepsilon^{3}
$$

and $\left.\left(\mathcal{U}_{0}^{\varepsilon}-\mathbf{z}^{\varepsilon}\right)\right|_{t=0}=0$. Then we repeat the calculations from the remaining part of the proof of Proposition 1 to get

$$
\left\|\partial_{t}\left(\mathcal{U}_{0}^{\varepsilon}-\mathbf{z}^{\varepsilon}\right)\right\|_{L^{2}\left(\Omega^{\varepsilon}\right)^{9}} \leq C \varepsilon^{2} .
$$

For the rest of the estimates, we just follow the proof of Proposition 2.

\section{Effective problem}

Let $\Sigma_{T}=\Sigma \times(0, T)$ and let

$$
\begin{gathered}
V^{\text {eff }}(\Omega)=\left\{\mathbf{b} \in H^{1}(\Omega)^{3}: \mathbf{b}=0 \text { on } \Sigma_{L}, b_{3}=0 \text { on } \Sigma,\right. \\
\left.\mathbf{b} \text { is L-periodic in }\left(x_{1}, x_{2}\right)\right\}, \\
\text { and } \mathcal{W}^{\text {eff }}=\left\{\varphi \in V^{e f f}(\Omega): \operatorname{div} \varphi=0 \text { in } \Omega\right\} .
\end{gathered}
$$

We consider the problem (27)-(32) which admits a solution $\mathbf{u}^{\text {eff }} \in L^{2}\left(0, T ; \mathcal{W}^{\text {eff }}\right)$, $\partial_{t} \mathbf{u}^{\text {eff }} \in L^{4 / 3}\left(0, T ;\left(\mathcal{W}^{\text {eff }}\right)^{\prime}\right)$, div $\mathbf{u}^{\text {eff }}=0$. Again $p^{\text {eff }}$ is determined following [41].

Our aim is to prove that there are smooth solutions to (27)-(32) close to $\mathbf{u}^{0}$. In this scope, we use a perturbation argument, which requires the hypothesis $(\mathbf{H 1})$ on $\mathbf{u}^{0}$. 
Proposition 6. Let us suppose the hypothesis (H1) and let $\mathbf{f} \in C^{\infty}(\bar{\Omega} \times[0, T])^{3}$, be such that supp $\mathbf{f} \subset \bar{\Omega} \times(0, T]$, and it is periodic in $\left(x_{1}, x_{2}\right)$ with period $L$. Then problem (27)-(32) has a solution $\left\{\mathbf{u}^{\text {eff }}, p^{\text {eff }}\right\} \in L^{2}\left(0, T ; \mathcal{W}^{\text {eff }} \cap H^{2}(\Omega)^{3}\right) \times L^{2}\left(0, T ; H^{1}(\Omega)\right)$, such that $\left\{\partial_{t} \mathbf{u}^{\text {eff }}, \partial_{t} p^{\text {eff }}\right\} \in L^{2}\left(0, T ; H^{1}(\Omega)^{3}\right) \times L^{2}\left(0, T ; L^{2}(\Omega)\right)$. $\left\{\mathbf{u}^{\text {eff }}, p^{\text {eff }}\right\}$ is unique.

Proof. We search for $\mathbf{u}^{\text {eff }}$ in the form $\mathbf{u}^{\text {eff }}=\mathbf{u}^{0}+\mathbf{w}$. We are looking for $\mathbf{w} \in$ $L^{2}\left(0, T ; \mathcal{W}^{e f f}\right)$, with $\partial_{t} \mathbf{w} \in L^{2}\left(0, T ; \mathcal{W}^{e f f}\right)$, and such that for all $\varphi \in \mathcal{W}^{\text {eff }}$, we have

$$
\begin{aligned}
& \int_{\Omega}\left(\frac{\partial \mathbf{w}}{\partial t}+\operatorname{div}\left(\mathbf{w} \otimes \mathbf{w}+\mathbf{w} \otimes \mathbf{u}^{0}+\mathbf{u}^{0} \otimes \mathbf{w}\right)\right) \varphi d x \\
& +\frac{1}{\operatorname{Re}} \int_{\Omega^{\varepsilon}} 2 D(\mathbf{w}): D(\varphi) d x-\frac{1}{\varepsilon \mathbf{R e}} \int_{\Sigma} M^{-1}\left(w_{1}, w_{2}\right) \cdot\left(\varphi_{1}, \varphi_{2}\right) d S \\
& =-\frac{1}{\operatorname{Re}} \int_{\Sigma} \frac{\partial}{\partial x_{3}}\left(u_{1}^{0}, u_{2}^{0}\right) \cdot\left(\varphi_{1}, \varphi_{2}\right) d S \\
& \mathbf{w}(x, 0)=0 \text { on } \Omega^{\varepsilon} .
\end{aligned}
$$

The classical way of proving existence of a solution for (87)-(88) is to construct an approximate solution, to get uniform a priori estimates, and then to use the compactness method for passing to the limit. This is the strategy for the Navier-Stokes equations with no-slip condition at the boundary and it works for this problem as well. Having ambition to pass to the zero viscosity limit requires construction of the special smooth basis. For the construction in 2D we refer to [18]. In 3D it is more complicated and we refer to work of Chen and Quin [19], see also [38]. Nevertheless, here we are concerned only by existence for fixed Reynolds number $\mathbf{R e}$ and an $H^{1}$-basis is enough. A problem related to (87)-(88) is studied in details in Section 3. Here, we just prove the a priori estimates; the rest of the proof is identical to the corresponding result from Section 3. Thus, we will manipulate $\mathbf{w}$ as a smooth function. This can be justified by manipulating its Galerkin approximation or by working with the smooth solution defined for a short time.

The proof of the a priori estimates will consist of several steps:

a) First, we insert $\mathbf{w}$ as a test function in (87) and get

$$
\begin{aligned}
& \frac{1}{2} \frac{d}{d t} \int_{\Omega}|\mathbf{w}(t)|^{2} d x+\frac{2}{\mathbf{R e}} \int_{\Omega}|D(\mathbf{w}(t))|^{2} d x+\int_{\Omega}(\mathbf{w}(t) \nabla) \mathbf{u}^{0} \mathbf{w}(t) d x \\
& -\frac{1}{\varepsilon \mathbf{R e}} \int_{\Sigma} M^{-1}\left(w_{1}, w_{2}\right) \cdot\left(w_{1}, w_{2}\right) d S=-\frac{1}{\mathbf{R e}} \int_{\Sigma} \frac{\partial}{\partial x_{3}}\left(u_{1}^{0}, u_{2}^{0}\right) \cdot\left(w_{1}, w_{2}\right) d S \\
& \leq C \varepsilon^{1 / 2}\left\|\frac{\left(w_{1}, w_{2}\right)(t)}{\sqrt{\varepsilon}}\right\|_{L^{2}\left(\Omega^{\varepsilon} \backslash \Omega\right)} .
\end{aligned}
$$

Using the fact that hypothesis (H1) yields $\mathbf{u}^{0} \in L^{2}\left(0, T ; H^{2}(\Omega)\right)$, we obtain for all $t \in(0, T)$

$$
\left|\int_{0}^{t} \int_{\Omega}(\mathbf{w}(\tau) \nabla) \mathbf{u}^{0}(\tau) \mathbf{w}(\tau) d x d \tau\right| \leq C|| \mathbf{u}^{0} \|_{L^{\infty}\left(Q_{T}\right)} \int_{0}^{t} \int_{\Omega}|\mathbf{w}(\tau)|^{2} d x d \tau .
$$

Using Gronwall's inequality we get now

$$
\|\mathbf{w}\|_{L^{\infty}\left(0, T ; L^{2}(\Omega)\right)} \leq C \sqrt{\varepsilon} .
$$

Next $\left|\int_{0}^{t} \int_{\Omega}(\mathbf{w}(\tau) \nabla) \mathbf{u}^{0}(\tau) \mathbf{w}(\tau) d x d \tau\right| \leq C \varepsilon$ and (89) implies

$$
\begin{aligned}
& \frac{1}{2} \frac{d}{d t} \int_{\Omega}|\mathbf{w}(t)|^{2} d x+\frac{2}{\operatorname{Re}} \int_{\Omega}|D(\mathbf{w}(t))|^{2} d x \\
& \leq C \varepsilon^{1 / 2}\|\nabla \mathbf{w}(t)\|_{L^{2}(\Omega)}+C \varepsilon
\end{aligned}
$$


Integrating in (89) with respect to t, and using Korn's inequality, we obtain the following estimates:

$$
\max _{0 \leq t \leq T}\|\mathbf{w}(t)\|_{L^{2}(\Omega)^{3}}^{2}+\int_{0}^{T}\|\nabla \mathbf{w}(t)\|_{L^{2}(\Omega)^{9}}^{2} d t+\frac{1}{\varepsilon} \int_{0}^{T}\|\mathbf{w}(t)\|_{L^{2}(\Sigma)^{3}}^{2} d t \leq C \varepsilon .
$$

Next, evaluating the weak formulation (87) at $t=0$, we obtain $\frac{\partial \mathbf{w}}{\partial t}(0)=0$.

b) In order to prove the estimates for the time derivative, the following estimate will be useful. We reconsider equation (87) tested with $\mathbf{w}$, and use estimate (92). We obtain the following estimate of $\|\nabla \mathbf{w}(t)\|_{L^{2}(\Omega)^{9}}^{2}$ for all $t$ from its smoothness interval

$$
\|\nabla \mathbf{w}(t)\|_{L^{2}(\Omega)^{9}}^{2} \leq c \sqrt{\varepsilon}\left(\sqrt{\varepsilon}+\left\|\partial_{t} \mathbf{w}(t)\right\|_{L^{2}\left(\Omega^{\varepsilon}\right)^{3}}\right) .
$$

c) Now, we start to prove the estimates for the time derivative. To this end, we consider the variational problem for $\partial_{t} \mathbf{w}$, which reads as follows:

$$
\begin{aligned}
& \int_{\Omega} \frac{\partial\left(\partial_{t} \mathbf{w}\right)}{\partial t} \varphi+\int_{\Omega} \operatorname{div}\left(\partial_{t} \mathbf{w} \otimes \mathbf{w}+\mathbf{w} \otimes \partial_{t} \mathbf{w}\right) \varphi d x \\
& +\int_{\Omega} \operatorname{div}\left(\partial_{t} \mathbf{w} \otimes \mathbf{u}^{0}+\mathbf{w} \otimes \partial_{t} \mathbf{u}^{0}+\partial_{t} \mathbf{u}^{0} \otimes \mathbf{w}+\mathbf{u}^{0} \otimes \partial_{t} \mathbf{w}\right) \varphi d x \\
& +\frac{1}{\operatorname{Re}} \int_{\Omega} 2 D\left(\partial_{t} \mathbf{w}\right): D(\varphi) d x-\frac{1}{\varepsilon \mathbf{R e}} \int_{\Sigma} M^{-1} \partial_{t}\left(w_{1}, w_{2}\right) \cdot\left(\varphi_{1}, \varphi_{2}\right) d \Sigma \\
& =-\frac{1}{\operatorname{Re}} \int_{\Sigma} \frac{\partial}{\partial x_{3}} \partial_{t}\left(u_{1}^{0}, u_{2}^{0}\right) \cdot\left(\varphi_{1}, \varphi_{2}\right) d S, \quad \forall \varphi \in \mathcal{W}^{e f f}, \\
& \partial_{t} \mathbf{w}(x, 0)=0 \quad \text { on } \Omega^{\varepsilon} .
\end{aligned}
$$

We test (94) with $\partial_{t} \mathbf{w}$ and estimate the terms. In difference with the system (87)-(88), when testing the problem (94)-(95) by $\partial_{t} \mathbf{w}$ the time derivative of the inertia term gives a contribution. Using estimate (93), the corresponding terms are estimated as follows. We begin with the estimate

$$
\begin{aligned}
& \left|\int_{\Omega}\left(\partial_{t} \mathbf{w} \nabla\right) \mathbf{w} \partial_{t} \mathbf{w}\right| \leq c\|\nabla \mathbf{w}(t)\|_{L^{2}(\Omega)^{9}}\left\|\nabla \partial_{t} \mathbf{w}(t)\right\|_{L^{2}(\Omega)^{9}}^{2} \\
& \leq c \varepsilon^{1 / 4}\left(\varepsilon^{1 / 4}+\left\|\partial_{t} \mathbf{w}(t)\right\|_{L^{2}(\Omega)^{3}}^{1 / 2}\right)\left\|\nabla \partial_{t} \mathbf{w}(t)\right\|_{L^{2}(\Omega)^{9}}^{2}
\end{aligned}
$$

The "Oseen like" terms behave slightly better. A typical estimate reads

$$
\begin{aligned}
& \left|\int_{\Omega}\left(\partial_{t} \mathbf{u}^{0} \nabla\right) \mathbf{w} \partial_{t} \mathbf{w}\right| \leq c\|\nabla \mathbf{w}(t)\|_{L^{2}(\Omega)^{9}}\left\|\nabla \partial_{t} \mathbf{w}(t)\right\|_{L^{2}(\Omega)^{9}}\left\|\nabla \partial_{t} \mathbf{u}^{0}(t)\right\|_{L^{2}(\Omega)^{9}} \\
& \leq c \varepsilon^{1 / 4}\left(\varepsilon^{1 / 4}+\left\|\partial_{t} \mathbf{w}(t)\right\|_{L^{2}(\Omega)^{3}}^{1 / 2}\right)\left\|\nabla \partial_{t} \mathbf{w}(t)\right\|_{L^{2}(\Omega)^{9}}\left\|\nabla \partial_{t} \mathbf{u}^{0}(t)\right\|_{L^{2}(\Omega)^{9}} .
\end{aligned}
$$

Inserting (96)-(97) into equation (94) tested by $\partial_{t} \mathbf{w}$, we obtain the following differential inequality

$$
\frac{1}{2} \frac{d}{d t} \int_{\Omega}\left|\partial_{t} \mathbf{w}(t)\right|^{2}+\left\|\nabla \partial_{t} \mathbf{w}(t)\right\|_{L^{2}(\Omega)^{9}}^{2}\left\{1-c \varepsilon^{1 / 4}\left\|\partial_{t} \mathbf{w}(t)\right\|_{L^{2}(\Omega)^{9}}^{1 / 2}\right\} \leq C \varepsilon
$$

Now for all $t$ from the smoothness interval and such that $\left\|\partial_{t} \mathbf{w}(t)\right\|_{L^{2}(\Omega)^{9}} \leq C \varepsilon^{-1 / 2}$, we have

$$
\left\|\partial_{t} \mathbf{w}(t)\right\|_{L^{2}(\Omega)^{9}}^{2} \leq C \varepsilon .
$$


Therefore the estimate (99) remains valid at the smoothness interval.

d) Finally, we see that the estimate (99) remain valid on $(0, T)$. These a priori estimates allow proving existence on standard way. By the obtained regularity, uniqueness is obvious.

Next we compare $\mathbf{u}^{\text {eff }}$ with the approximation calculated in Section 4 . Let us set

$$
\mathbf{v}^{0}=\mathbf{u}^{0}-\varepsilon \sum_{j, k=1}^{2} M_{k j} \mathbf{g}^{k j} ; \quad p_{v}=p^{0}-\varepsilon \sum_{j, k=1}^{2} M_{k j} p_{g}^{k j} .
$$

Then $\left\{\mathbf{v}^{0}, p_{v}\right\}$ satisfies the system

$$
\begin{gathered}
\frac{\partial \mathbf{v}^{0}}{\partial t}+\operatorname{div}\left(\mathbf{v}^{0} \otimes \mathbf{v}^{0}\right)-\frac{1}{\mathbf{R e}} \operatorname{div}\left(2 D\left(\mathbf{v}^{0}\right)-p_{v} I\right)= \\
\varepsilon^{2} \sum_{k, j, l, r=1}^{2} M_{k j} M_{l r} \operatorname{div}\left(\mathbf{g}^{k j} \otimes \mathbf{g}^{l r}\right) \text { in } Q_{T}, \\
\mathbf{v}^{0}=0 \quad \text { on } \Sigma_{L} \times(0, T), \\
v_{3}^{0}=0 \text { and } v_{i}^{0}=-\varepsilon \sum_{j=1}^{2} M_{i j} \frac{\partial}{\partial x_{3}} v_{j}^{0}+\varepsilon^{2} \sum_{k, j, r=1}^{2} M_{i j} M_{k r} \frac{\partial}{\partial x_{3}} g_{j}^{k r}, i=1,2, \quad \text { on } \Sigma_{T}, \\
\mathbf{v}^{0} \quad \text { is periodic in }\left(x_{1}, x_{2}\right) \text { with period } L, \\
\left.\mathbf{v}^{0}\right|_{t=0}=0 \quad \text { on } \Omega .
\end{gathered}
$$

In complete analogy with the proof of Proposition 5 , we have

Proposition 7. We have

$$
\begin{gathered}
\int_{0}^{T}\left\|\nabla\left(\mathbf{u}^{e f f}-\mathbf{u}^{0}+\varepsilon \sum_{j, k=1}^{2} M_{k j} \mathbf{g}^{k j}\right)(t)\right\|_{L^{2}(\Omega)^{9}}^{2} d t+ \\
\int_{0}^{T}\left\|\nabla \partial_{t}\left(\mathbf{u}^{e f f}-\mathbf{u}^{0}+\varepsilon \sum_{j, k=1}^{2} M_{k j} \mathbf{g}^{k j}\right)(t)\right\|_{L^{2}(\Omega)^{9}}^{2} d t \leq C \varepsilon^{3} \\
\max _{0 \leq t \leq T}\left(\left\|\left(\mathbf{u}^{e f f}-\mathbf{u}^{0}+\varepsilon \sum_{j, k=1}^{2} M_{k j} \mathbf{g}^{k j}\right)(t)\right\|_{L^{2}(\Omega)^{3}}^{2}+\right. \\
\left.\left\|\partial_{t}\left(\mathbf{u}^{e f f}-\mathbf{u}^{0}+\varepsilon \sum_{j, k=1}^{2} M_{k j} \mathbf{g}^{k j}\right)(t)\right\|_{L^{2}(\Omega)^{3}}^{2}\right) \leq C \varepsilon^{3} \\
\int_{0}^{T}\left\|\left(p^{e f f}-p^{0}+\varepsilon \sum_{j, k=1}^{2} M_{k j} p_{g}^{k j}\right)(t)\right\|_{L^{2}\left(\Omega_{l o c}\right)^{3}}^{2} d t \leq C \varepsilon^{3} .
\end{gathered}
$$

Now we are in situation to prove Theorem 2

Proof. (of Theorem 2). First estimate (77) implies

$$
\| \nabla\left(\mathbf{u}^{\varepsilon}-\left(\mathbf{u}^{0}-\varepsilon \sum_{j, k=1}^{2} M_{k j} \mathbf{g}^{k j}\right) \|_{H^{1}\left(0, T ; L^{1}(\Omega)\right)} \leq C \varepsilon .\right.
$$


Then the above estimate together with (106) implies (33). Next we use estimates (77)-(81) to conclude that

$$
\left\|\mathbf{u}^{\varepsilon}-\left(\mathbf{u}^{0}-\varepsilon \sum_{j, k=1}^{2} M_{k j} \mathbf{g}^{k j}\right)\right\|_{H^{1}\left(0, T ; L^{2}(\Sigma)\right)} \leq C \varepsilon^{3 / 2}
$$

and we use the very weak solutions to the Oseen problem to conclude (34). (35) follows from the equation and the preceding estimates.

\section{Appendix: Very weak solutions to a nonstationary Os- een system in $\Omega$}

Let $\mathbf{a} \in L^{r}\left(0, T ; L^{q}(\Omega)^{3}\right)$ for $q \in(3, \infty]$, and $r \in[2, \infty), 1 / r+3 /(2 q) \leq 1 / 2$. Let $\mathbf{G}_{1} \in L^{2}\left(Q_{T}\right)^{3}, G_{2} \in L^{2}\left(Q_{T}\right)^{9}$, and $\xi \in L^{2}\left(\Sigma_{T}\right)^{3}$. We consider the following Oseen system in $Q_{T}=\Omega \times(0, T)$ :

$$
\left\{\begin{array}{l}
\frac{\partial \mathbf{b}}{\partial t}+\operatorname{div}(\mathbf{a} \otimes \mathbf{b}+\mathbf{b} \otimes \mathbf{a}) \\
-\frac{1}{\mathbf{R e}} \operatorname{div}(2 D(\mathbf{b})-P I)=\mathbf{G}_{1}+\operatorname{div} G_{2} \text { in } Q_{T} \\
\operatorname{div} \mathbf{b}=0 \quad \text { in } Q_{T} ; \\
\mathbf{b}(x, 0)=0 \quad \text { in } \Omega ; \\
\mathbf{b}=\xi \text { on } \Sigma_{T}=\left(\Sigma \cup \Sigma_{L}\right) \times(0, T) \\
\{\mathbf{b}, P\}, \text { is L-periodic in }\left(x_{1}, x_{2}\right)
\end{array}\right.
$$

Our aim is to show the existence of a weak solution $(\mathbf{b}, P) \in L^{2}\left(Q_{T}\right)^{3} \times H^{-1}\left(Q_{T}\right)$ for (109). To this end, we use essentially the transposition method from [20].

Thus, let us test problem (109) by a smooth test function $(\boldsymbol{\Phi}, \pi)$, satisfying $\boldsymbol{\Phi}(x, T)=$ 0 in $\Omega, \boldsymbol{\Phi}$ is $L$-periodic in $\left(x_{1}, x_{2}\right)$, and $\boldsymbol{\Phi}=0$ on $\Sigma_{T}$. Furthermore, $\pi$ is $L$-periodic in $\left(x_{1}, x_{2}\right)$. We get

$$
\begin{aligned}
& \int_{0}^{T}<\mathbf{G}_{1}+\operatorname{div} G_{2}, \mathbf{\Phi}>d t=\int_{0}^{T}<\frac{\partial \mathbf{b}}{\partial t}+\operatorname{div}(\mathbf{a} \otimes \mathbf{b}+\mathbf{b} \otimes \mathbf{a}) \\
& -\frac{1}{\mathbf{R e}} \operatorname{div}(2 D(\mathbf{b})-P I), \boldsymbol{\Phi}>d t=-\frac{1}{\operatorname{Re}} \int_{Q_{T}} P \operatorname{div} \mathbf{\Phi} d x d t+ \\
& \frac{1}{\mathbf{R e}} \int_{\Sigma_{T}}(2 D(\mathbf{\Phi})-\pi I) \nu \xi d S d t+\int_{Q_{T}} \mathbf{b} \cdot\left(-\frac{\partial \mathbf{\Phi}}{\partial t}-2 D(\mathbf{\Phi}) \mathbf{a}-\right. \\
& \left.\frac{1}{\mathbf{R e}} \operatorname{div}(2 D(\boldsymbol{\Phi})-\pi I)\right) d x d t .
\end{aligned}
$$

We define the space

$$
\mathcal{H}=\left\{z \in H_{0}^{1}\left(Q_{T}\right), \int_{\Omega} z d x=0\right\},
$$


and denote by $\mathcal{H}^{*}$ its dual. For $(\mathbf{g}, s) \in L^{2}\left(Q_{T}\right)^{3} \times \mathcal{H}$, let now $\{\boldsymbol{\Phi}, \pi\}$ be given by

$$
\left\{\begin{array}{l}
-\frac{\partial \boldsymbol{\Phi}}{\partial t}-2 D(\boldsymbol{\Phi}) \mathbf{a}-\frac{1}{\mathbf{R e}} \operatorname{div}(2 D(\boldsymbol{\Phi})-\pi I)=\mathbf{g} \text { in } Q_{T} \\
\operatorname{div} \mathbf{\Phi}=s \text { in } Q_{T} \\
\phi=0, \text { on } \Sigma_{T} ; \boldsymbol{\Phi}(x, T)=0 \text { in } \Omega \\
\{\boldsymbol{\Phi}, \pi\} \text { is L-periodic in }\left(x_{1}, x_{2}\right) .
\end{array}\right.
$$

After Theorem $1^{1}$, page 86 in [31], for $(\mathrm{g}, s) \in L^{2}\left(Q_{T}\right)^{3} \times \mathcal{H}$, we have

$$
\boldsymbol{\Phi} \in L^{2}\left(0, T ; H^{2}(\Omega)^{3}\right), \frac{\partial \boldsymbol{\Phi}}{\partial t} \in L^{2}\left(Q_{T}\right)^{3}, \pi \in L^{2}\left(0, T ; H^{1}(\Omega)\right)
$$

with $\int_{\Sigma} \pi=0$, and the following estimates hold

$$
\int_{0}^{T}\|\boldsymbol{\Phi}\|_{H^{2}(\Omega)^{3}} d t+\int_{0}^{T}\|\nabla \pi\|_{L^{2}(\Omega)} d t \leq C\left(\|\mathbf{g}\|_{L^{2}\left(Q_{T}\right)^{3}}+\|\nabla s\|_{L^{2}\left(Q_{T}\right)}\right) .
$$

Now, analogously to the approach in [20] where the stationary Stokes system was treated, we consider the linear form

$$
\ell(\mathbf{g}, s)=\int_{0}^{T}\left\langle\mathbf{G}_{1}+\operatorname{div} G_{2}, \mathbf{\Phi}\right\rangle d t-\frac{1}{\mathbf{R e}} \int_{\Sigma_{T}}(2 D(\mathbf{\Phi})-\pi I) \nu \xi d S d t
$$

where $(\boldsymbol{\Phi}, \pi)$ is given by (111). Since $(\boldsymbol{\Phi}, \pi)$ satisfies (112), the linear form $\ell: L^{2}\left(Q_{T}\right)^{3} \times$ $\mathcal{H} \rightarrow \mathbb{R}$ is continuous, and we set

Definition 1. (A very weak variational formulation for the nonstationary Oseen problem (109)). $\{\mathbf{b}, P\}$ is a very weak solution for the problem (109) if

$$
\{\mathbf{b}, P\} \in L^{2}\left(Q_{T}\right)^{3} \times \mathcal{H}^{*}
$$

and satisfies

$$
\int_{0}^{T} \int_{\Omega} \mathbf{b} \cdot \mathbf{g} d x d t-\langle P, s\rangle_{\mathcal{H}^{*}, \mathcal{H}}=\ell(\mathbf{g}, s), \quad \forall \mathbf{g} \in L^{2}\left(Q_{T}\right)^{3}, \forall s \in \mathcal{H} .
$$

Because of the linearity and continuity of $\ell$, Riesz's theorem implies

Proposition 8. There exists a unique very weak solution $\{\mathbf{b}, P\}$ for (109). It satisfies the following estimates

$$
\|\mathbf{b}\|_{L^{2}\left(Q_{T}\right)^{3}} \leq c\left(Q_{T}\right)\left\{\left\|\mathbf{G}_{1}\right\|_{L^{2}\left(0, T ; L^{1}(\Omega)\right)^{3}}+\left\|G_{2}\right\|_{L^{2}\left(0, T ; L^{6 / 5}(\Omega)\right)^{9}}+\|\xi\|_{L^{2}\left(\Sigma_{T}\right)^{3}}\right\} .
$$

For more detailed theory of the very weak solutions for the Navier-Stokes equations we refer to [22].

\footnotetext{
${ }^{1}$ In fact the result is explicitly stated only in the 2nd Russian edition, Ladyzhenskaya, O.A., Matematičeskie vopros'i dinamiki vjaskoi nesžimaemoi židkosti, Nauka, Moscow, 1970, as Theorem 1', page 110. Getting the needed result from Theorem 1 from [31], page 86, requires some additional work.
} 


\section{References}

[1] Y. Achdou, O. Pironneau, F. Valentin, Shape control versus boundary control, eds F.Murat et al, Equations aux dérivées partielles et applications. Articles dédiés à J.L.Lions, Elsevier, Paris, 1998, p. 1-18.

[2] Y. Achdou, O. Pironneau, F. Valentin, Effective Boundary Conditions for Laminar Flows over Periodic Rough Boundaries, J. Comp. Phys. , 147 (1998), p. 187-218.

[3] G. Allaire, M. Amar, Boundary layer tails in periodic homogenization, ESAIM : Control, Optimisation and Calculus of Variations 4(1999), p. 209-243.

[4] Y. Amirat, J. Simon, Influence de la rugosité en hydrodynamique laminaire, C. R. Acad. Sci. Paris, Série I, 323 (1996), p. 313-318.

[5] Y. Amirat, J. Simon, Riblet and Drag Minimization, in Cox, S (ed) et al., Optimization methods in PDEs, Contemp. Math, 209, p.9-17, American Math. Soc., Providence, 1997.

[6] Y. Amirat, D. Bresch, J. Lemoine, J. Simon, Effect of rugosity on a flow governed by stationary Navier-Stokes equations, Quart. Appl. Math. 59 (2001), no. 4, 769785 .

[7] Y. Amirat, O. Bodart, U. de Maio, A. Gaudiello Effective boundary condition for Stokes flow over a very rough surface J. of Differential Equations 254 (2013), 3395-3430.

[8] A. Basson, D. Gérard-Varet, Wall laws for fluid flows at a boundary with random roughness, Comm. Pure Appl. Math. 61 (2008), no. 7, 941-987.

[9] D. Bresch, D. Gérard-Varet, Roughness-induced effects on the quasi-geostrophic model, Comm. Math. Phys. 253 (2005), no. 1, 81-119.

[10] M. Bonnivard, D. Bucur, The uniform rugorosity effect, J. Math. Fluid Mech. 14, (2012), $201-215$.

[11] D. Bucur, E. Feireisl, Š. Nečasová, Influence of wall roughness on the slip behavior of viscous fluids, Proc. Royal Soc. Edinburgh, 138A (2008), 957-973.

[12] D. Bucur, E. Feireisl, Š. Nečasová, and J. Wolf. On the asymptotic limit of the Navier-Stokes system on domains with rough boundaries. J. Differential Equations, 244:2890-2908, 2008.

[13] D. Bucur, E. Feireisl, Š. Nečasová, Boundary behavior of viscous fluids: Influence of wall roughness and friction-driven boundary conditions, Arch. Ration. Mech. Anal. 197 (2010), no. 1, 117-138.

[14] T. Carraro, C. Goll, A. Marciniak-Czochra and A. Mikelić, Pressure jump interface law for the Stokes-Darcy coupling: Confirmation by direct numerical simulations, preprint arXiv:1301.6580 [math.NA], 2013.

[15] J. Casado-Díaz,M. Luna-Laynez, F. J. Suárez-Grau, On the Navier boundary condition for viscous fluids in rough domains. SeMA J. No. 58 (2012), 5 - 24 
[16] J. Casado-Díaz, M. Luna-Laynez, F. J. Suárez-Grau, Asymptotic behavior of a viscous fluid near a rough boundary. BAIL 2010 - boundary and interior layers, computational and asymptotic methods, 57-64, Lect. Notes Comput. Sci. Eng., 81, Springer, Heidelberg, 2011.

[17] J. Casado-Díaz, E. Fernández-Cara, and J. Simon. Why viscous fluids adhere to rugose walls: A mathematical explanation. J. Differential Equations, 189:526-537, 2003.

[18] Th. Clopeau, A.Mikelić, R.Robert, On the vanishing viscosity limit for the $2 D$ incompressible Navier-Stokes equations with the friction type boundary conditions, Nonlinearity, Vol. 11 (1998), no. 6, 1625-1636.

[19] Chen, Gui-Qiang, Qian, Zhongmin, A study of the Navier-Stokes equations with the kinematic and Navier boundary conditions. Indiana Univ. Math. J. 59 (2010), no. $2,721-760$.

[20] C. Conca, Étude d'un fluide traversant une paroi perforée I. Comportement limite près de la paroi, J. Math. pures et appl., 66, (1987), pp. 1-44, II. Comportement limite loin de la paroi, J. Math. pures et appl., 66, (1987), pp. 45-69.

[21] A-L. Dalibard, D. Gérard-Varet, Effective boundary condition at a rough surface starting from a slip condition Journal of Differential Equations 251, 3297-3658 (2011).

[22] Farwig, Reinhard; Galdi, Giovanni P.; Sohr, Hermann, A new class of weak solutions of the Navier-Stokes equations with nonhomogeneous data, J. Math. Fluid Mech., 8 (2006), no. 3, 423-444.

[23] D. Gérard-Varet, The Navier wall law at a boundary with random roughness, Comm. Math. Phys. 286 (2009), no. 1, 81-110.

[24] D. Gérard-Varet, N. Masmoudi, Relevance of the slip condition for fluid flows near an irregular boundary Comm. Math. Phys. 295, 99-137 (2010).

[25] W. Jäger, A. Mikelić , On the Boundary Conditions at the Contact Interface between a Porous Medium and a Free Fluid, Annali della Scuola Normale Superiore di Pisa, Classe Fisiche e Matematiche - Serie IV 23 (1996), Fasc. 3, 403-465.

[26] W. Jäger, A. Mikelić, On the effective equations for a viscous incompressible fluid flow through a filter of finite thickness, Communications on Pure and Applied Mathematics 51 (1998), 1073-1121.

[27] W. Jäger, A. Mikelić, On the interface boundary conditions by Beavers, Joseph and Saffman, SIAM J. Appl. Math., 60 (2000), p. 1111-1127.

[28] W. Jäger, A. Mikelić, On the roughness-induced effective boundary conditions for a viscous flow, J. Differential Equations 170 (2001), p. 96-122.

[29] W. Jäger, A. Mikelić, N. Neuß, Asymptotic analysis of the laminar viscous flow over a porous bed, SIAM J. Sci. Comput. 22 (2001), p. 2006 - 2028.

[30] W. Jäger, A. Mikelić, Couette Flows over a Rough Boundary and Drag Reduction, Comm. Math. Phys. 232 (2003), p. 429-455. 
[31] Ladyzhenskaya, O.A., The Mathematical Theory of Viscous Incompressible Flow, Gordon and Breach, 2nd edition, New York, 1969.

[32] J. L. Lions, Some Methods in the Mathematical Analysis of Systems and Their Control, Gordon and Breach, New York, 1981.

[33] P. Luchini, Asymptotic analysis of laminar boundary-layer flow over finely grooved surfaces, Eur. J. Mech. B/Fluids , 14 (1995), p. 169 - 195.

[34] A. Marciniak-Czochra, A. Mikelić, Effective pressure interface law for transport phenomena between an unconfined fluid and a porous medium using homogenization, SIAM: Multiscale modeling and simulation, Vol. 10, No. 2 (2012), p. 285-305.

[35] A. Mikelić, Rough boundaries and wall laws, in "Qualitative properties of solutions to partial differential equations", Lecture notes of Necas Center for mathematical modeling, edited by E. Feireisl, P. Kaplický and J. Málek, Vol. 5, p. 103 - 134, Matfyzpress, Publishing House of the Faculty of Mathematics and Physics Charles University in Prague, Prague, 2009.

[36] B. Mohammadi, O. Pironneau, F. Valentin, Rough Boundaries and Wall Laws, Int. J. Numer. Meth. Fluids, 27 (1998), p. 169-177.

[37] C. L. M. H. Navier, Sur les lois de l'équilibre et du mouvement des corps élastiques, Mem. Acad. R. Sci. Inst. France, 369 (1827).

[38] J. Neustupa,P. Penel, Approximation of a Solution to the Euler Equation by Solutions of the Navier - Stokes Equation. J. Math. Fluid Mech. 15 (2013), no. 1, 179 - 196.

[39] R. L. Panton, Incompressible Flow , John Wiley and Sons, New York, 1984.

[40] N. V. Priezjev and S.M. Troian. Influence of periodic wall roughness on the slip behaviour at liquid/solid interfaces: molecular versus continuum predictions. $J$. Fluid Mech., 554:25-46, 2006.

[41] Taniuchi, S., On generalized energy inequality of the Navier-Stokes equations. Manuscripta Math. 69,237-254 (1990).

[42] Temam, R., Navier Stokes equations. North Holland, Amsterdam (1977). 